Article

\title{
Experimental Studies of Lubricant Flow and Friction in Partially Filled Gaps
}

\author{
Michael Müller *(D), Lukas Stahl $(\mathbb{D}$ and Georg-Peter Ostermeyer \\ Institut für Dynamik und Schwingungen, Technische Universität Braunschweig, 38106 Braunschweig, Germany; \\ l.stahl@tu-braunschweig.de (L.S.); gp.ostermeyer@tu-bs.de (G.-P.O.) \\ * Correspondence: mi.mueller@tu-bs.de; Tel.: +49-(0)531-391-7005
}

Received: 30 October 2018; Accepted: 12 December 2018; Published: 18 December 2018

\begin{abstract}
Starved lubrication is an important strategy for minimizing the amount of lubricant needed, and also inevitably occurs during idling and fail-safe lubrication. In this regime, however, the flow of the lubricant and the related friction coefficients are yet to be fully understood. This research aims to make fundamental contributions to the understanding of contact mechanics of partially lubricated contacts. Recent experiments with a pin-on-disk tribometer examined the microscopic behavior of partially filled gaps. Using a new experimental setup on a macroscale, new insights into partially filled gaps with rough surfaces were gained. This work presents the systematic analyses of the lubricant flow, friction coefficients, and other variables over a wide range of friction parameters. Distinct friction behaviors were observed, and similar effects occur on both the micro and macroscale. The experimental results show that a typical Stribeck characteristic is visible regarding not only the relative velocity, but also regarding the lubricant filling level in the gap. The fluid exhibits a variety of flow patterns for various velocities and viscosities. The patterns relate to different friction regimes, such as dry friction and mixed lubrication. It is concluded that the filling level is a valid parameter for regulating the transition from dry friction to hydrodynamic lubrication. These findings are quantified regarding the filling level and it is shown that for the identification of the friction regimes the filling level is an independent parameter in addition to the established parameters like speed, viscosity and pressure.
\end{abstract}

Keywords: starved lubrication; partially filled gap; lubricated; flow patterns; coefficient of friction

\section{Introduction}

Friction is an omnipresent effect that significantly influences every technical system. In some cases, friction is an essential operating principle of the system, for example in brakes. Usually, however, friction and wear are undesired occurrences, for example in gears and bearings. A variety of approaches are available for minimizing friction and wear within a system. These range from magnetic gearings to classic lubrication methods. Conventional lubrication systems apply a lubricant in the contact zone that builds up a hydrodynamic pressure between the contact surfaces. This pressure is able to transmit the contact forces and therefore separate the solid surfaces, thereby greatly reducing friction, wear and noise [1].

Popular lubrication strategies ensure a maximized amount of lubricant within the contact area. This is beneficial with respect to operational safety and longevity of a machine, but comes with great drawbacks regarding cost efficiency, constructional demands and ecological influences. Therefore, various strategies have been designed to minimize the amount of lubricant needed. Furthermore, idling and lubricant supply failure can reduce the fluid amount in the gap. This can lead to a state in which the gap between the two contact partners is not entirely filled with lubricant, known as a partially filled gap. Additionally, partially filled gaps occur when a dry contact is influenced by, 
for example, environmentally induced fluid wetting. Currently, the contact and fluid dynamics in such a gap are yet to be fully understood. The present studies address the flow and the related friction of partially filled contacts $[1,2]$.

In order to classify the partially filled lubrication within the broader context of lubrication, the Neo-Stribeck-Curve can be considered [3]. Figure 1 shows the coefficient of friction (COF) over a non-dimensional parameter consisting of the viscosity, the rotational speed and the pressure of the contact [1]. The COF exhibits a variety of behaviors and has a constant value in the regime of boundary friction. The regime of starved lubrication with partially filled gaps occurs between the mixed and boundary lubrication regimes. In this regime, friction and wear are highly sensitive to small variations in friction parameters. While this behavior is well documented based on experiments [2,3], a comprehensive understanding of the transition from a dry gap to partially lubricated state has not yet been achieved. To this end, a variety of approaches have been undertaken. Early experiments and simulation regarding the starved lubrication regime of a ball bearing were carried out in [4]. Starved lubrication during piston ring lubrication was examined in [5]. In [6], starved lubrication for thrust journals was studied. In [7], the first solution for a numerical program with partially filled gaps is presented. The authors of the present work have also developed a numerical model for the simulation of partially filled gaps [8-10]. In [11], starved lubrication in a wet clutch is examined. The work focuses on the oil flow in correlation to the speed of the clutch and considers influences such as centrifugal forces.

However, all these references emphasize the results and effects of starved lubrication and little is known about the basic contact mechanism that takes place in partially filled gaps with rough surfaces. Therefore, in the present work, an experimental approach towards investigating partially filled gaps with rough surfaces is presented.

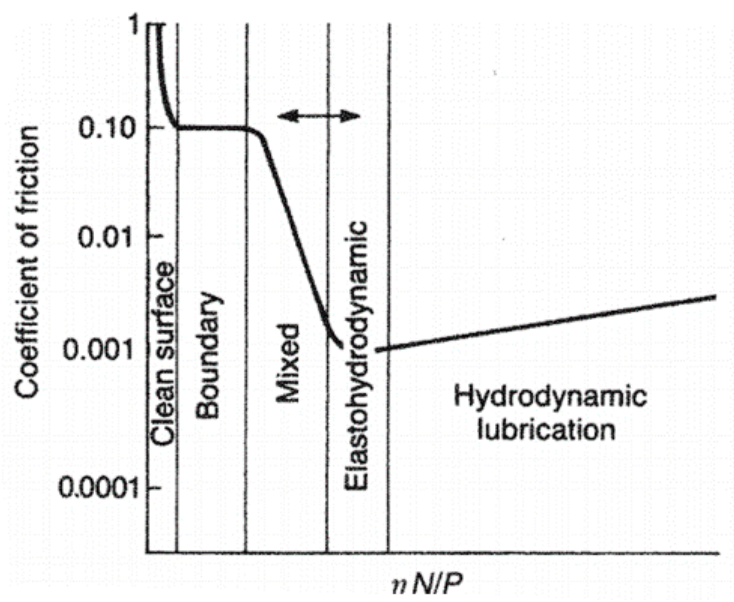

Figure 1. Stribeck curve with starved lubrication between the boundary and mixed lubrication (reprinted from [1]).

Commonly, the non-dimensional parameter of Figure 1 represents the entirety of the lubricated friction parameters in the Stribeck context. Regarding the Stribeck curve, the friction regime is typically determined by a load parameter such as the velocity or the normal force. It is obvious that the amount of fluid significantly effects the friction regime. However, there is very little explicit research on the influence of the fluid volume in the gap. The amount of fluid in the gap is commonly represented with the filling level $\theta$ that is defined by the percentage of local fluid volume related to the local gap volume. The filling level is furthermore used to evaluate the flow in the starvation region, for example by scaling the viscosity of the flow with $\eta=\theta \cdot \eta_{\text {fluid }}$ [12]. With the present systematic study, an improved understanding of the influence of the filling level is sought.

The experiments feature the variation of fluid volume within the gap. This procedure was used to evaluate the hypothesis that the friction regime can also be controlled via the filling level and to 
quantify the influence of the fluid volume. Dry friction is a friction regime in which no fluid is present in the contact and thus the normal force is transmitted via solid body contact. Adding even small amounts of lubricant leads to drastic changes in the friction regime. Fluid particles can build up localized hydrodynamic pressure on a molecular scale $[13,14]$. When the fluid volume in the gap increases, additional effects such as cavitation must be considered. Experimental results for these scenarios can be found in [15-17]. The effects of partially filled gaps will be sequentially further investigated with respect to the coefficient of friction and the lubricant flow.

A first set of experiments were carried out using a conventional tribometer setup. This research was published in [18] and will be shortly summarized here. The High Load Tribometer (HLT) experiments were used to gain a first understanding of the relevant fluid volumes for this setup, rather than to carry out a full systematic study. Therefore, the HLT experiments only consist of one test series and continuative tests are planned in the near future.

The basic experimental concept on the HLT consists of a pin on disk setup that enables the measurement of the COF [19]. Figures 2 and 3 present the corresponding experimental equipment. The upper picture shows the full tribometer and some of the measurement equipment. Below this, the cast iron disk and the steel pin are presented in detail. The setup shown is a typical example of a classic technical contact. Figure $3 a$ shows the friction track on the grey cast iron disk and Figure $3 b$ shows a sample pin $(10 \mathrm{~mm} \times 20 \mathrm{~mm})$ topography in a run in state.

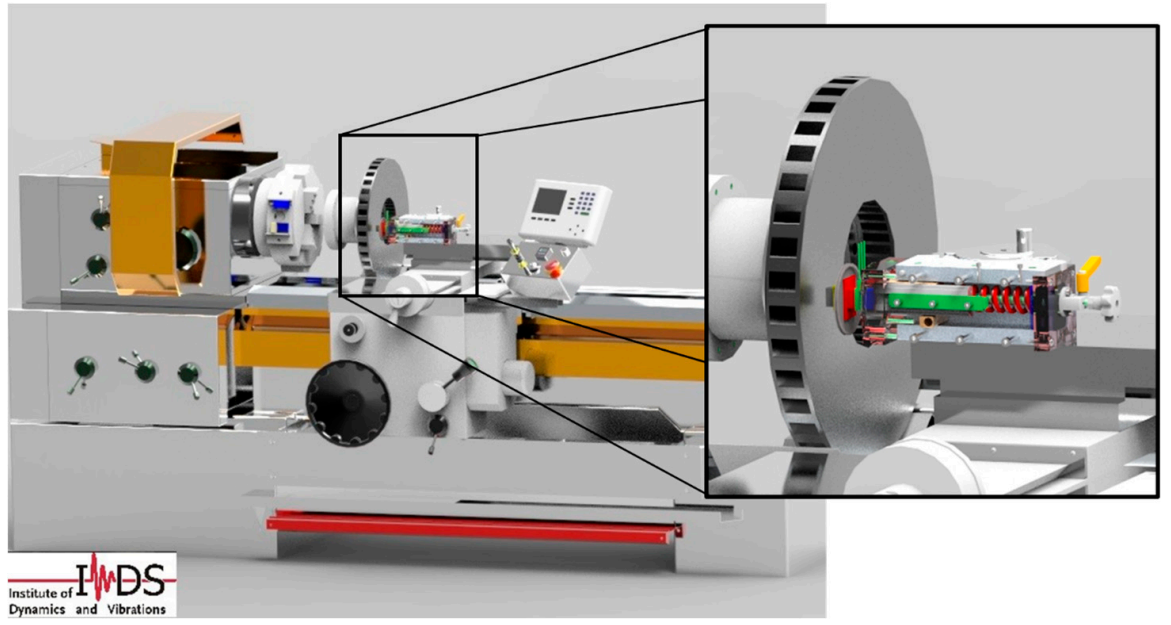

Figure 2. Overview of the tribometer with detail of the contact zone.

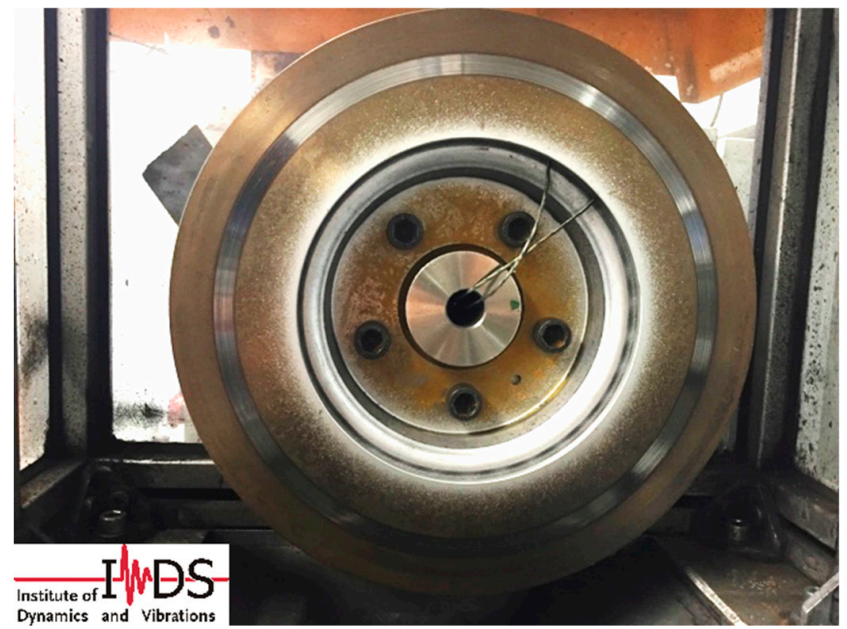

(a)

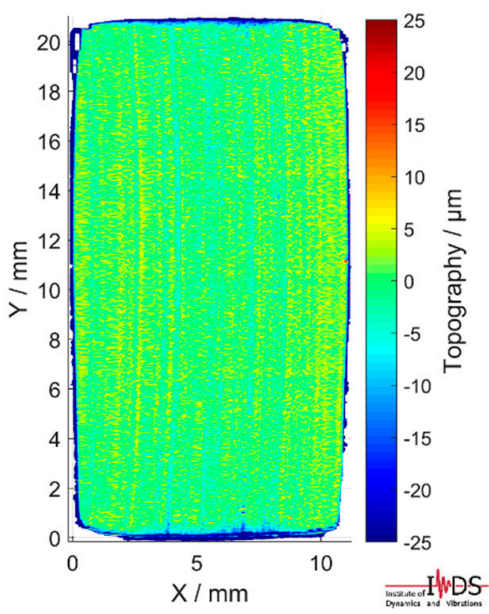

(b)

Figure 3. Friction partners: (a) brake disc; (b) pin. 
The studies show that the application of small amounts of lubricant massively influence the contact processes. The first measurement was carried out with a dry contact, followed by three lubricated applications. Afterwards, a final measurement was carried out after cleaning the contact. The amount of lubricant applied was varied. A precision syringe was used to apply drops of the lubricant. One drop is equivalent to about $12 \mu \mathrm{L}$ of fluid volume. The lubricant used was glycerol, which was chosen for its high viscosity. Figure 4 shows the coefficient of friction for the described procedure.

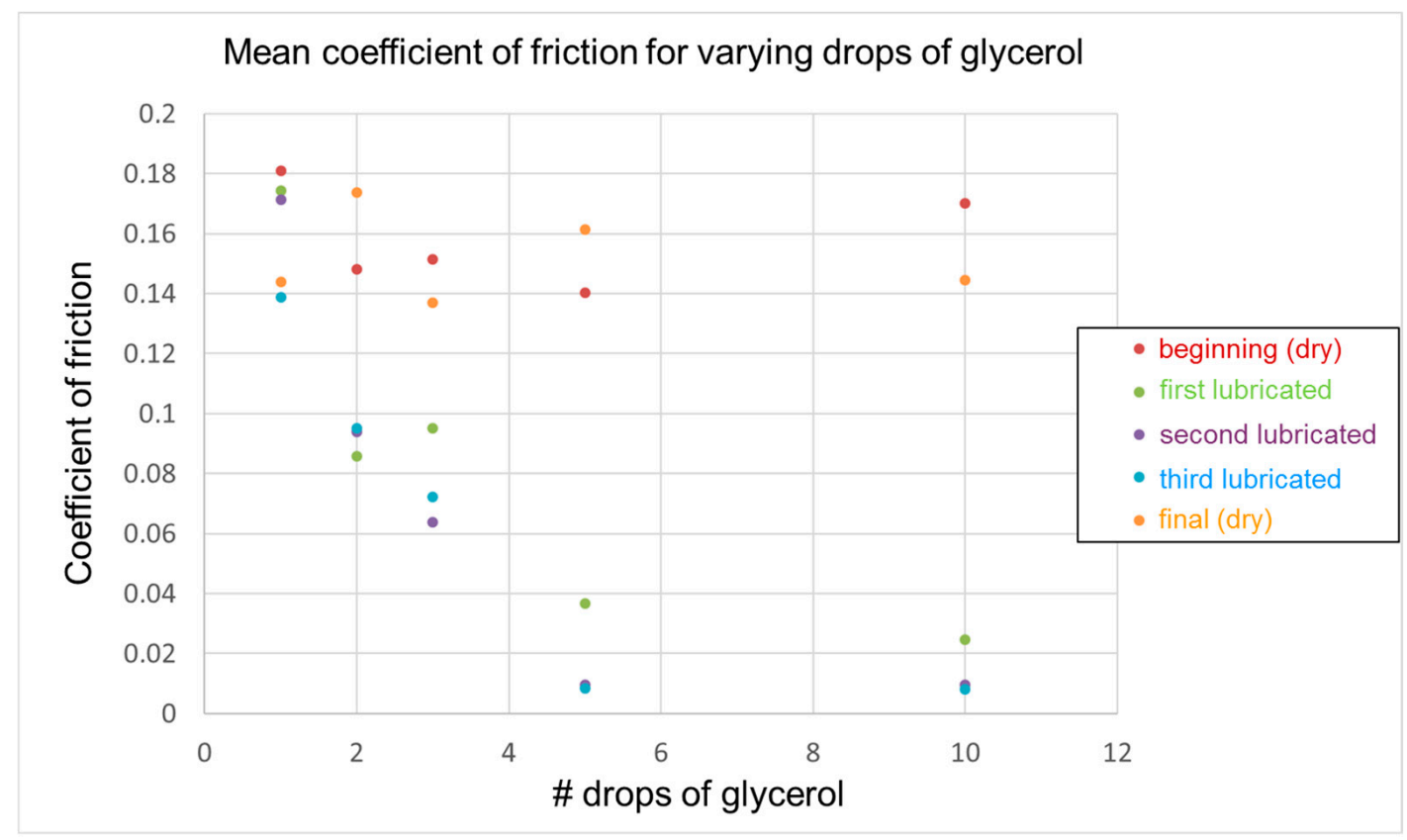

Figure 4. Coefficient of friction (COF) over fluid amount of glycerol, HLT-tribometer [18].

The red and orange dots show the COF for the dry measurements. The COF is in the range of $0.14-0.18$. When adding two drops of lubricant (this corresponds to about $24 \mu \mathrm{L}$ of fluid), the $\mathrm{COF}$ is already cut in half. Adding five drops leads to a COF as low as 0.01. Increasing the fluid volume further does not further affect the COF. This leads to the assumption that the gap is fully filled with lubricant and that in this regime the contact is mainly influenced by hydrodynamic friction [18]. This supports the thesis that, within boundaries, the transition from dry to lubricated friction can also be controlled with the lubricant volume. The sensitivity of the COF to the fluid volume illustrates the need to understand the friction principles during this transition. While it seems obvious that the fluid volume effects the friction regime, there is little known about the quantification of the fluid influence. The processes during starvation have been mostly studied for single asperity contact (for example [4]). The study of the fundamental processes of the fluid behavior in partially filled gaps with rough surfaces requires new experimental approaches, as conventional pin-on-disc set-ups (such as the HLT) are not suitable for this purpose.

\section{Experiments and Methods}

\subsection{Experimental Setup}

The HLT experiments revealed the influence of the lubricant amount in tribological contacts. Interpretation of these results is limited, however, due to the constraints of this experiment. Due to the solid surfaces of the contacting materials, the fluid cannot be well observed within the contact, and only integral forces can be measured during the procedure. Essential aspects such as the fluid flow cannot be sufficiently addressed. 
In order to understand more aspects of partially filled contacts, the contact problem was investigated using a different experimental setup with a macroscopic friction scenario. For this experiment, the surface roughness and the overall gap heights are typically in the range of $\mathrm{mm}$. The scalability between the test scenarios is discussed in detail later.

The new experiment aims to offer an improved understanding of the processes in contacts with partially filled gaps and the corresponding lubricant flows. This approach then aims to transfer the findings to microscopic contacts. For the transfer, a variety of requirements have to be met, as discussed below. Table 1 shows a comparison of the fundamental properties of microscale and macroscale contacts in terms of experimental studies.

Table 1. Qualitative characteristics of macroscopic (Wear Debris Investigator (WDI)) and microscopic contacts (HLT).

\begin{tabular}{ccc}
\hline Characteristic & Microscopic Contacts (HLT) & Macroscopic Contacts (WDI) \\
\hline Roughnesses & range $\mu \mathrm{m}$ & range mm \\
\hline Tribologically relevant? & yes & no \\
\hline Number of asperities & very high & very low \\
\hline Wear & significant & mostly negligible \\
\hline Observability of forces & $\begin{array}{c}\text { integral normal force } \\
\text { integral friction force }\end{array}$ & spatially distributed normal and friction force \\
\hline Observability of the fluid flow & not possible & possible \\
\hline
\end{tabular}

The two scales have different surface roughness's. The HLT contacts are tribologically relevant, and can be found in technical applications. They have a high number of asperities that interact during mixed or dry friction. Therefore, only integral forces can be measured. However, the contact of the WDI has only a small number of asperities that allow for spatially distributed measurement. The wear at the HLT is significant and influences the surface topography which has to be considered when interpreting the experiment results. Wear occurs also during Wear Debris Investigator (WDI) experiments, however, due to the macroscopic roughness the wear does not change the surface topography significantly. Finally, only the macroscopic contact allows the observation of the flow and the corresponding friction behavior.

For this reason, the new experiments were carried out using the WDI [20]. This new test stand was originally developed to investigate the behavior of particles in a dry friction contact. Details of its technical equipment and first results can be found in [20]. The WDI is a rotational tribometer that measures a variety of output variables. Most notably, the tribological contact consists of two glass disks that enable the unobstructed observation of the contact area. Figure 5 shows an overview of the WDI. Figure 6 shows a detailed view of the contact area. 


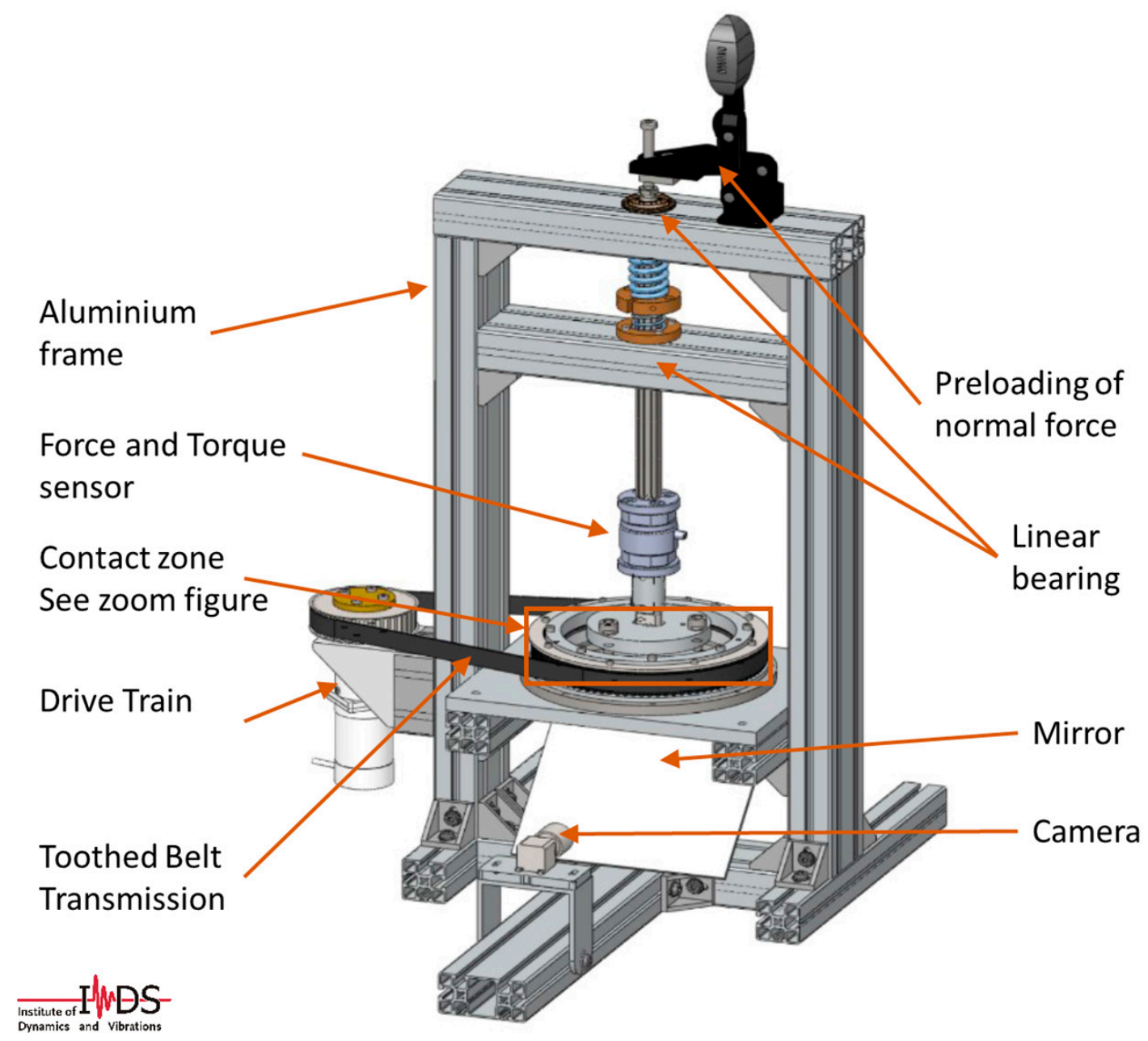

Figure 5. Overview on the WDI [20].

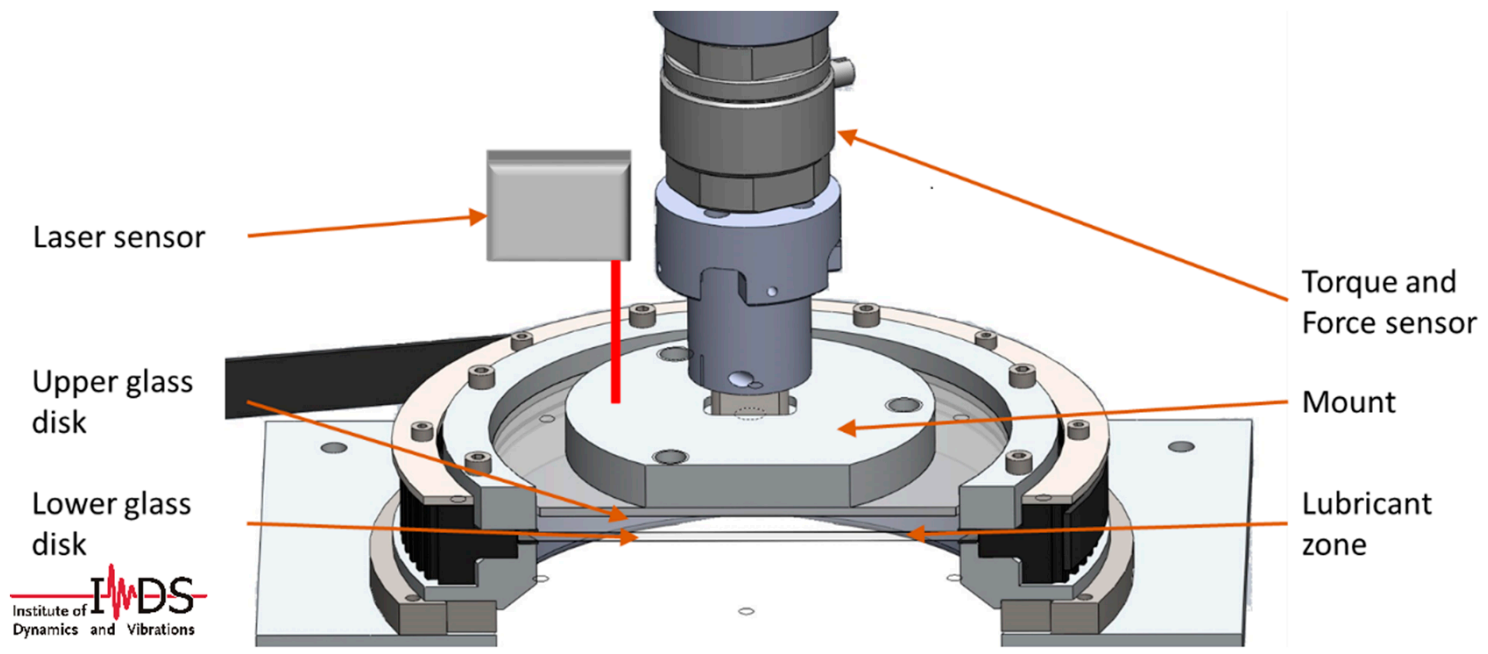

Figure 6. Contact zone of WDI [20].

The tribological contact is in the center of the WDI and consists of two glass disks. The lower disk can be rotated via an electric drive within the range of 0 to $60 \mathrm{rpm}$. The upper disk is rotationally fixed and can be loaded onto the lower disk with varying normal forces. The mount of the upper disk features a torque and force sensor that monitors the contact forces. The contact zone itself is visible through a mirror on the bottom that is also used for recording the contact zone with a high-speed 
camera. Additionally, a laser distance sensor measures the height of the upper disk. This can be used as a reference for the overall gap height.

The two disks of the tribological contact are made of ornamental glass. Due to its transparency, the glass enables observation of the contact area. The patterned and structured surface of the glass creates the tribological topography of the friction contact. The ornamental glass is available in various types and standards with a wide range of topographies, therefore enabling a great variety of contact gaps.

In the presented study, the patterned glasses Ornament 523 Monumental M weiß $4 \mathrm{~mm}$ (referenced as 523R) and Ornament 528 Monumental S weiß $4 \mathrm{~mm}$ (528R) were used. To observe the fluid flow in partially filled gaps, one contact partner must be transparent and its roughness should be higher than common technical surfaces by magnitudes of order. The ornamental glass was chosen for its comparability to a technical surface in terms of the ratio of the asperity length to the asperity height. For the glass, the length of an asperity is in the range of $\mathrm{cm}$ while the height of an asperity is in the range of $\mathrm{mm}$. Additionally, the glass has little wear. The surface topographies were analyzed and assessed regarding their suitability for the contact. The surfaces were classified by their average surface roughness. Figure 7 shows a sample disc of pattern 523R, and Figure 8 shows the measured surface topography of the two glasses used for the study.

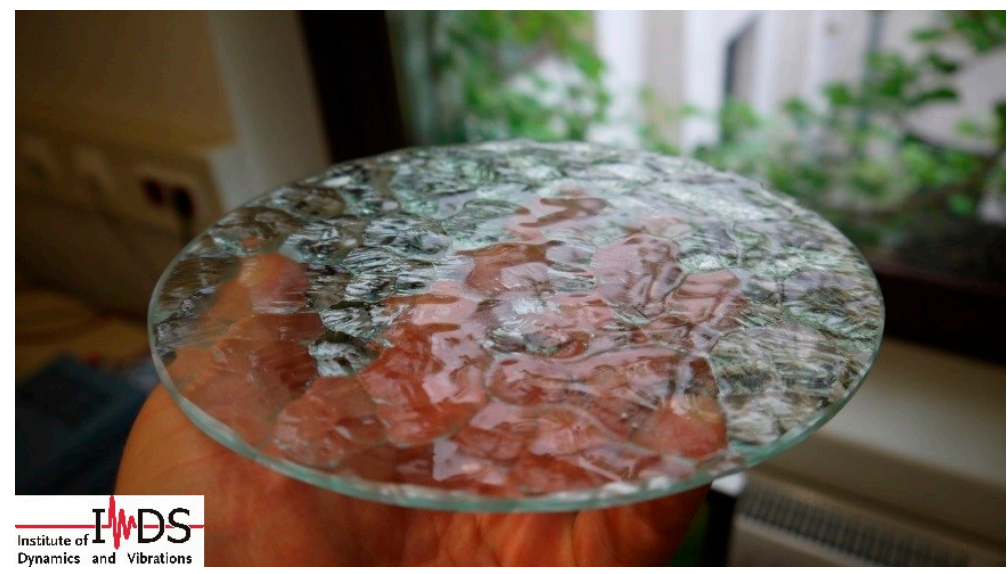

Figure 7. View of the ornamental glass 523R.

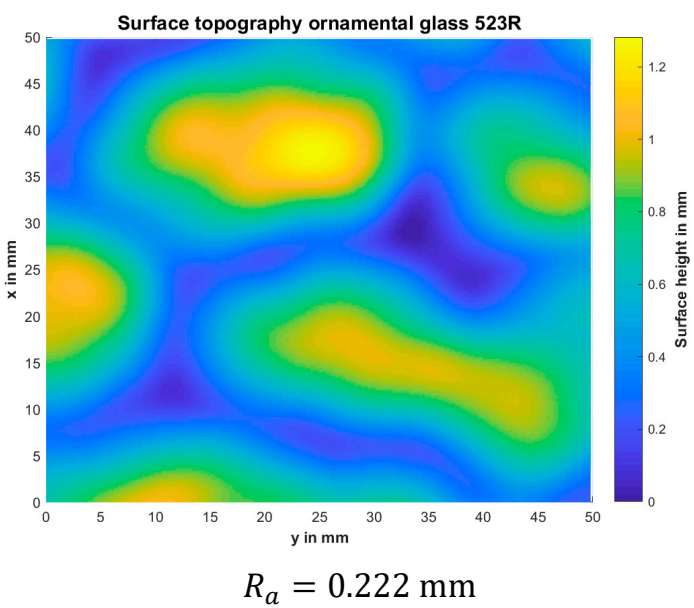

(a)

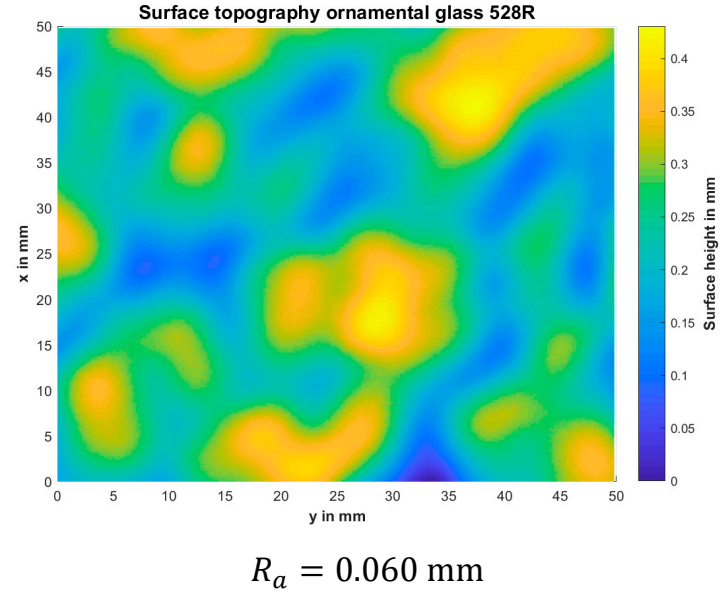

(b)

Figure 8. Measured topography of ornamental glass (section): (a) 523R, (b) 528R. 
The arithmetic surface roughness's of 528R and 523R are $0.060 \mathrm{~mm}$ and $0.222 \mathrm{~mm}$ respectively. In continuation of the HLT experiments, standard glycerol was chosen as the lubricant for the macroscale experiments. Under normal conditions, glycerol has a viscosity of about $1 \mathrm{~Pa} \cdot \mathrm{s}$. With varying mixtures of water and glycerol, a variation of the fluid viscosity was achieved [21].

The measurement procedure of the macroscale experiments includes the variation of four parameters: Relative velocity, global filling level, topography and lubricant viscosity. The relative velocity was controlled via the rotational speed of the disk. The filling level was modified by the initially applied amount of lubricant in the gap. The fluid volume was manually applied onto the contact using a high precision syringe. To apply the fluid, the upper disk was raised. No leakage was detected during the test, so it was assumed that the fluid volume was constant. During a measurement procedure, first, a variation of the rotational speed was carried out, after which the smallest amount of fluid was added (see Figure 9). Next, the speed was varied for every new amount of fluid. After the measurement set with the greatest amount of fluid (fully filled state), the WDI was disassembled and cleaned. Then, the entire procedure was repeated a minimum of five times for statistical purposes. The topography was varied by using different ornamental glasses and the viscosity was varied by using different glycerol/water ratios. The full procedure can be seen in Figure 9.

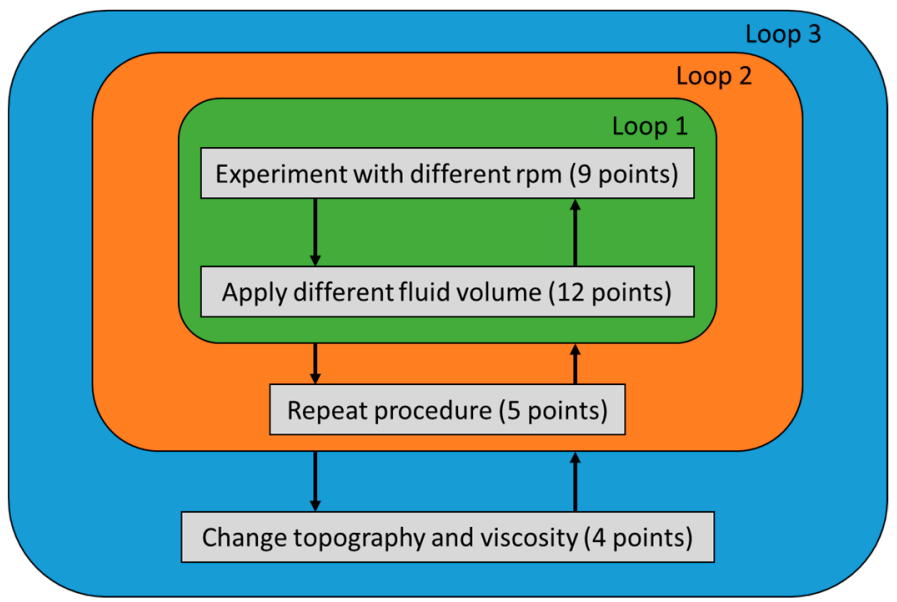

Figure 9. Full measurement procedure for the macroscale experiments (WDI).

The measurement setup produces a variety of different output signals: The normal force and the rotational friction torque of the upper disks, the engine rotational speed and electric current, the height of the upper disk and a time stamp. Figure 10 shows an exemplary section of the normal force and the friction torque over time.

The normal force fluctuates around $30 \mathrm{~N}$, which is the static load of the contact caused by the dead weight of the upper disk and mount. The positive axis of the friction torque is in the opposite direction of the movement of the lower disk. The figure shows that the amplitude of the measurement signal is highly variable. This is related to the small number of asperities in the contact zone. The peaks in the signal are the result of individual asperity interactions. In some cases, the signal's features can be visually associated with specific topographic conditions. Overall, high peaks in both signals occur in an interrelated manner-during instances of high normal force, the friction torque also rises. A series of pretests suggested that the variations in the measurement signal do not originate from eigenmodes, but are in fact caused by the contact itself. The first eigenfrequency in normal direction is at around $80 \mathrm{~Hz}$ which is far greater than the dynamics of this contact. Additionally, the influence of inertias was examined with a model. It was shown that such influences are small and that the measured signals are very close to the actual friction forces. This work focuses on the integral aspects of the contact. For this purpose, all signals were averaged and only integer revolutions were taken into further consideration. 

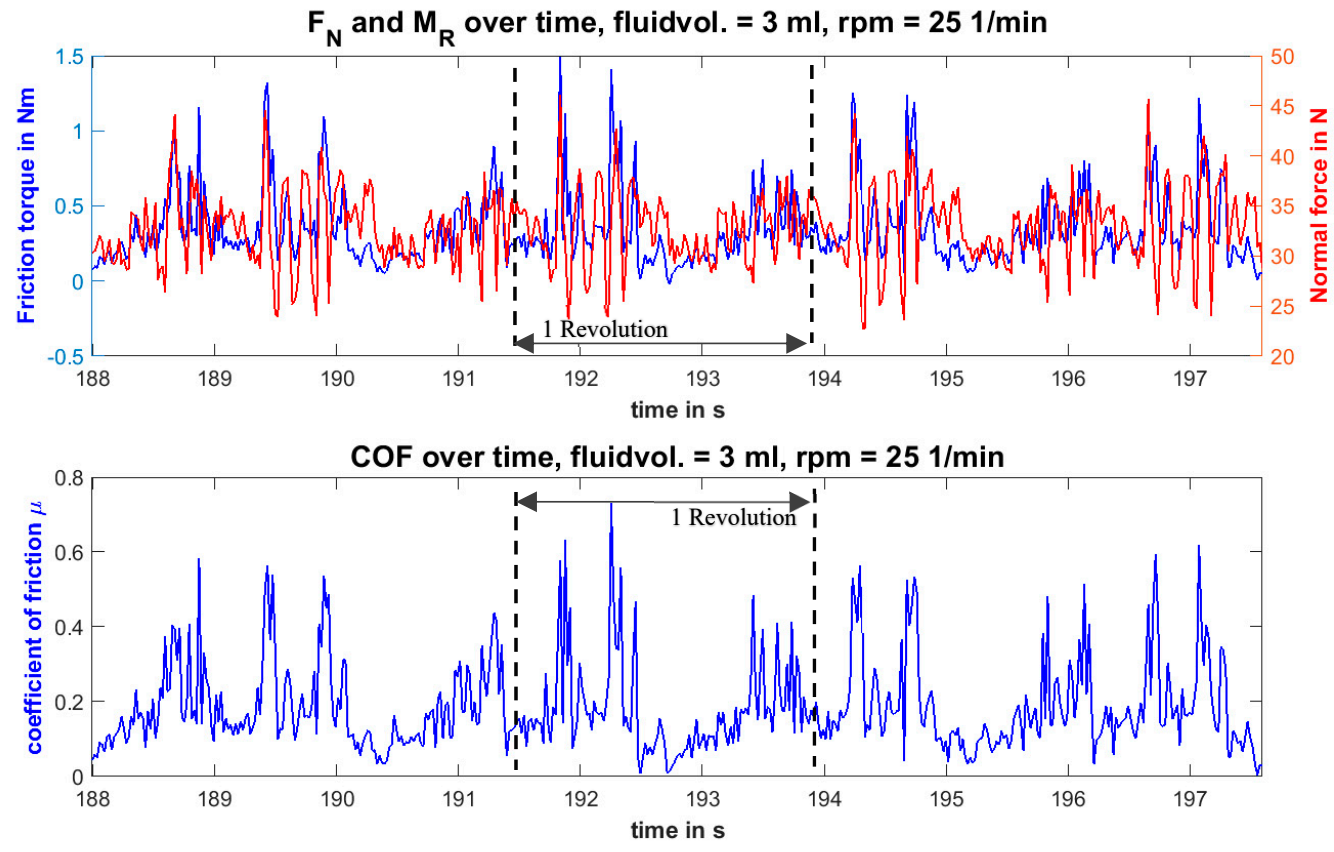

Figure 10. Sample of measurement data: normal force and friction torque over time at a glyc. volume of $3 \mathrm{~mL}$ and at $25 \mathrm{rpm}$.

With the average data, the COF $\mu$ was computed using $\mu=\frac{\tau_{R}}{p_{N}}$ where $\tau_{R}$ is the radial shear stress and $p_{N}$ the nominal pressure. The shear stress can be calculated by solving an integral over the entire disk area using the known friction torque with $M_{R}=\int_{0}^{R} \int_{0}^{2 \pi} \tau_{R} \cdot r^{2} \mathrm{~d} \varphi \mathrm{d} r$. Assuming $\mu(x, y)=$ const and $p(x, y)=$ const this results in $\mu=\frac{M_{R}}{\frac{2}{3} \cdot R \cdot F_{N}}$. These assumptions are a significant simplification, however, for this application they allow for a qualitative evaluation.

Additionally, the vertical kinematics of the upper disk were measured using a laser triangulator. This information was used to gain further insights into the gap height and its dynamic behavior during the measurement procedure. Figure 11 shows the height evolution for a full measurement procedure. The movement of the upper disk is highly dynamic and caused by the macroscopic nature of the measurement. The variance of the disk height is considerable, but this vertical dynamic is still significantly smaller than the rotational movement of the fluid (comparing average circumference in the range of $0.25 \mathrm{~m}$ ). It is possible, however, to evaluate the relative change in the gap height, as presented in Section 3.3.

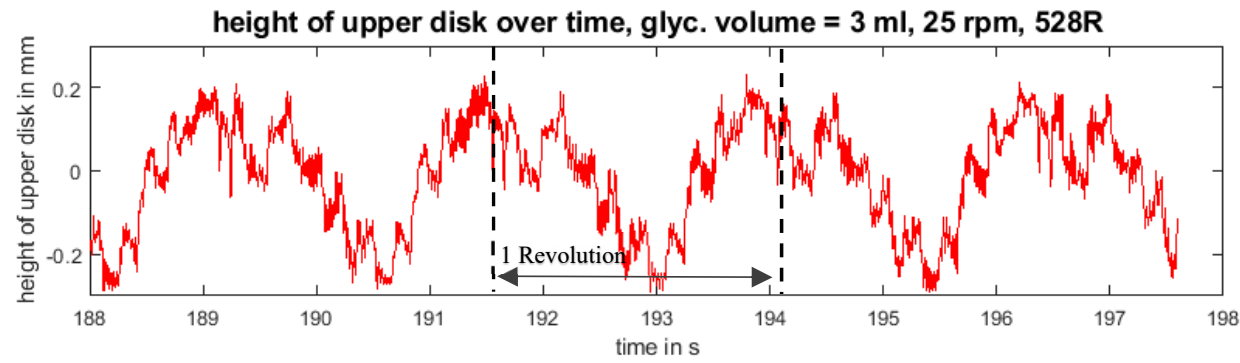

Figure 11. Height of the upper disk over time at a fluid volume of $0.4 \mathrm{~mL}$ and at $25 \mathrm{rpm}, 523 \mathrm{R}$.

The measured height was used to calculate the average rise in gap height for multiple speeds. This value represents the average rise in mean gap height compared to the mean gap height at $1 \mathrm{rpm}$. To calculate this value, the height of the upper disk was first averaged over integer revolutions for all velocities and filling levels. Second, subtracting the height at $1 \mathrm{rpm}$ from the dynamic height led to the average rise in gap height, as evaluated in Section 3.3. 


\subsection{Considerations Regarding Scalability}

The following section analyzes the admissibility of the WDI experiments. The experimental results in Section 3 imply that varying friction regimes occur during the tests. The results alone, however, might not be sufficient to prove that a hydrodynamic friction regime was achieved during HLT and WDI experiments. To validate the experimental results and to assess the comparability between the HLT and WDI experiments, this section contains preliminary considerations regarding scalability. Whether or not the Reynolds Equation was valid for the two experiments was examined. The validity was primarily treated as an indicator whether effects like convective fluid flow or solid body contact are significant.

When modelling technical lubrication problems, the Reynolds Equation is commonly used [22], shown here in the one dimensional form.

$$
\frac{\partial}{\partial x}\left(\frac{\rho h^{3}}{12 \eta} \cdot \frac{\partial p}{\partial x}\right)=u_{m} \cdot \frac{\partial}{\partial x}(h \rho)+\frac{\partial}{\partial t}(h \rho) .
$$

The derivation of the Reynolds Equation is scale independent and is applied to many problems of significantly different scale. The Reynolds Equation models lubrication in small bearings as well as in geophysical faults [23]. This leads to the assumption that characteristics of a lubricated problem are similar across different scales. With this hypothesis in mind, the new set of experiments was carried out on the WDI, that has a much larger gap height and roughness characteristic than prior investigated contacts.

To validate this hypothesis, the nature of a dimensionless Reynolds Equation and the scalability of fluid flow were investigated. Most approaches consider the derivation of the Reynolds Equation from the Navier-Stokes-Equations. A variety of simplifications were implemented to reduce the complexity of the Navier-Stokes-Equation. In order to verify the validity of the Reynolds Equation these simplifications were reviewed.

First, the Reynolds Equation assumes a laminar fluid flow. The state of the flow is commonly assessed with the Reynolds Number, which relates the inertial forces to the viscous forces. Generally, a flow with a Reynolds Number $R e<2300$ is considered as a laminar flow.

$$
R e=\frac{2 \rho v h_{0}}{\eta} .
$$

Second, the Reynolds Equation assumes that the viscous force in the direction of the gap is far greater than the viscous forces in the directions within the friction plane. However, it is known that the inertial forces become significant when the gap height rises. To evaluate this, the Modified Reynolds Number has been introduced in the literature [24-26]. This number explicitly compares the viscous force of the gap fluid shear with the inertial forces. For this comparison, a Modified Reynolds Number $R e^{*}$ less than one indicates that the Reynolds Equation is valid [26].

$$
R e^{*}=\frac{\rho v h_{0}^{2}}{L \eta}
$$

The Modified Reynolds Number was used to assess whether the Reynolds Equation is valid for the experiments. If the Reynolds Equation is valid, the influence of effects like convective fluid flow is not significant during the experiments. Lastly, the nature of the hydrodynamic lubrication was assessed by evaluating a dimensionless Reynolds Equation. The following procedure is commonly applied within engineering and fluid mechanics, and similar approaches can be found in [23-25,27-29].

Figure 12 shows a sample of a typical friction problem with a fully filled gap. The upper topography is moved while the lower one is immobile. The figure shows a characteristic section with the length $L$ and periodic boundaries. The gap topography can be described with the parameters shown. The Reynolds Equation can be simplified for the shown problem. The transient term $\frac{\partial}{\partial t}(h \rho)$ 
can be calculated with assumptions such as constant density and the known movement of the upper topography. Assuming a horizontal movement of the upper topography, the transient term can be transformed into a spatial derivative $\left(\frac{\partial}{\partial x}\right)$ and merged with the existing Couette term as shown in Equation (4).

$$
\frac{\partial}{\partial x}\left(\frac{h^{3}}{12 \eta} \cdot \frac{\partial p}{\partial x}\right)=\frac{u_{0}}{2} \frac{\partial}{\partial x}\left(h_{2}-h_{1}\right)=\frac{u_{0}}{2} \frac{\partial \bar{h}}{\partial x} .
$$

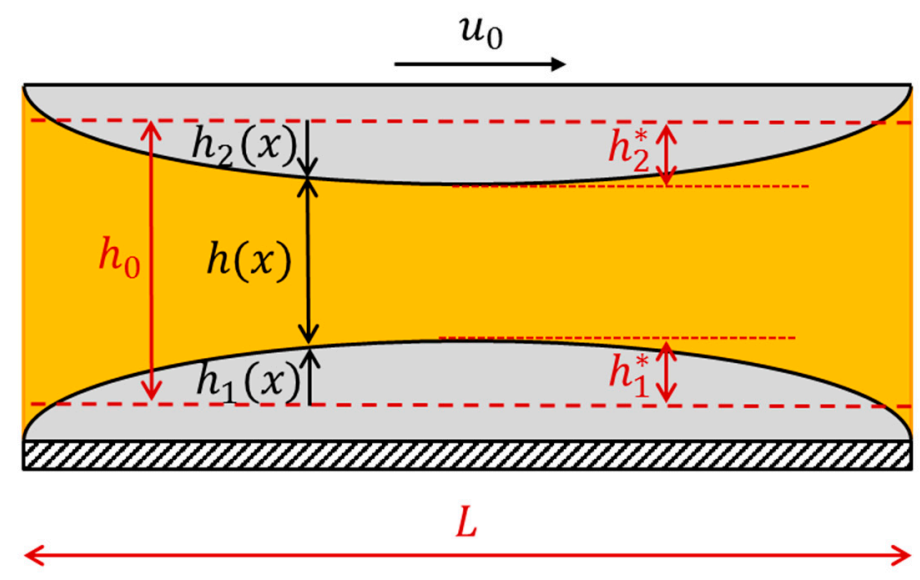

Figure 12. Example of a lubricated problem.

By introducing normalized variables into the Reynolds Equation, the equation becomes dimensionless. This approach is commonly used [23,24,29].

$$
X=\frac{x}{L} \quad H=\frac{h}{h_{0}} \quad P=\frac{p}{p_{0}} \quad \bar{H}=\frac{\bar{h}}{h^{*}}
$$

The variable $x$ is normalized with the parameters shown in Table 2 and the variance of the contact topography $\bar{h}$ is normalized with the topography parameter $h^{*} . h^{*}$ is a characteristic parameter of the contact topography and may be appropriately chosen to represent the given topography. In the following, $h^{*}$ will be defined as the sum of the mean arithmetic surface roughness. With this transformation, the Reynolds Equation can be rewritten in the form of Equation (6).

$$
\frac{h_{0}^{3} p_{0}}{6 L \eta u_{0} h^{*}} \frac{\partial}{\partial X}\left(H^{3} \frac{\partial P}{\partial X}\right)=\frac{\partial \bar{H}}{\partial X} .
$$

Table 2. Symbol directory.

\begin{tabular}{ccc}
\hline Symbol & Measure & Unit of Measure \\
\hline$\rho$ & Density & $\frac{\mathrm{kg}}{\mathrm{m}^{3}}$ \\
$v$ & Fluid velocity & $\frac{\mathrm{m}}{\mathrm{s}}$ \\
$h_{0}$ & Mean Gap height & $\mathrm{m}$ \\
$h$ & Gap height & $\mathrm{ma} \cdot \mathrm{s}$ \\
$\eta$ & Fluid viscosity & $\mathrm{m}$ \\
$L$ & Characteristic Length & $\mathrm{Pa}$ \\
$p$ & Pressure & $\mathrm{Pa}$ \\
$p_{0}$ & Mean Pressure & \\
\hline
\end{tabular}

In this form, the equation is separated into a normalization factor that is followed by a dimensionless differential equation. The normalization factor itself is also dimensionless and contains all information regarding the system and its parameters. This factor is now isolated and called $N$. 


$$
N=\frac{h_{0}^{3} p_{0}}{6 L \eta u_{0} h^{*}} .
$$

In the following, the normalization factor $N$ is used as a parameter that describes the state of the hydrodynamic lubrication. Mathematically, two lubrication problems with the same factor $N$ yield the same solution with the dimensionless Reynolds Equation (within boundaries). Therefore, for such problems, the results of the Reynolds Equation may be scaled using $N$, instead of computing the solution with the original Reynolds Equation. This approach is commonly used (compare $[23,24,26]$ ) and is only valid for hydrodynamic lubrication. The factors described will be used to assess the characteristics of the experiments.

First, simple simulations of the hydrodynamic friction regime have been accomplished using a lubrication model that can also include elastic deformations and solid body contact. These simulations were based on the scenario of Figure 12. Obviously, for these simulations the factor $N$ is the same for a wide variety of parameter configurations. For configurations where the gap height $h_{0}$ is greater than the roughness parameter $h^{*}, N$ is at 0.25 . A reduction of the gap height towards the solid body contact generates significantly higher values of $N$, indicating that the Reynolds Equation is no longer valid. The value of $N$ can thus be understood as an indicator of when hydrodynamics are present for a certain setup (here rough surfaces) and when mixed friction begins. For this purpose, $N$ serves as an indicator, with the help of which an estimation of the friction regime in experiments is possible. It is expected that values of $N$ in the range of 0.25 indicate that hydrodynamic friction is dominant, while higher values of $N$ imply that mixed friction occurs. Furthermore, it is expected that a comparison between similar friction systems (for example regarding the topography) with similar $N$-values is possible within certain limits.

In the following, the parameters are calculated for the experiments. Table 3 shows the estimated parameters of the factors for the experiments on the micro and macroscales at a hydrodynamic friction regime (fully filled).

Table 3. Parameters of HLT and WDI experiments.

\begin{tabular}{ccc}
\hline Symbol & HLT (Microscale) & WDI (Macroscale) \\
\hline$p_{0}$ & $250,000 \mathrm{~Pa}$ & $1350 \mathrm{~Pa}$ \\
$h_{0}$ & in the range of $10 \mu \mathrm{m}$ & $1.4 \mathrm{~mm}$ \\
$u_{0}$ & $1.0472 \frac{\mathrm{m}}{\mathrm{s}}$ & $0.352 \frac{\mathrm{m}}{\mathrm{s}}$ \\
$\eta$ & $1 \mathrm{~Pa} \cdot \mathrm{s}$ & $1 \mathrm{~Pa} \cdot \mathrm{s}$ \\
$h^{*}=2 \cdot R_{a}$ & in the range of $8 \mu \mathrm{m}$ & $440 \mu \mathrm{m}$ \\
$L$ & $20 \mu \mathrm{m}$ & $20 \mathrm{~mm}$ \\
\hline
\end{tabular}

The mean pressure was calculated using the applied pressure and the friction area. The mean gap height of the WDI was estimated based on the known topography (compare Figure 8) combined with the vertical movement of the upper glass. It was possible to calculate the overall gap height based on the volume between the two topographies. On the HLT disk, the lubricant forms a visible circular ring that spreads evenly on the friction track. The overall fluid volume of this ring is known. As a first approximation, the mean gap height for the HLT was estimated by calculating the height of this fluid film. The velocity of the upper topography was calculated using the known geometry and rotational speed. Due to the extensive rotational contact area of the WDI the relative speed of the upper disk is dependent on the radius. The velocity of the WDI was therefore calculated with the effective radius of $2 / 3$ the maximum (outer) radius.

The fluid was the same for both applications. No notable heating was detected during the short loading periods. The parameter $h^{*}$ is associated with the roughness of the topography. To allow a simple comparison between the topographies, the mean arithmetic surface roughness was chosen. The roughness was doubled to account for the two rough surfaces used. The topographies of the friction 
partners were measured (compare Figure 8) and the roughness' were calculated. The topographies at the HLT exhibit a strong orientation and there are visible radial grooves caused by the rotational motion. Here, the roughness in the direction of the flow was calculated (compare Figure 3) to comply with the model of Figure 12. Finally, the characteristic length was chosen to be the mean spacing between the asperities in the direction of the flow. Table 4 shows the factors for the two experiments (according to Equations (2), (3) and (7)).

Table 4. Non-dimensional factors for HLT and WDI experiments.

\begin{tabular}{ccc}
\hline Factor & HLT (Microscale) & WDI (Macroscale) \\
\hline$R e=\frac{2 \rho v h_{0}}{\eta}$ & 0.0126 & 0.5911 \\
$\operatorname{Re} e^{*} \approx \frac{\rho v h_{0}^{2}}{L \eta}$ & 0.0063 & 0.0414 \\
$N=\frac{h_{0}^{3} p_{0}}{6 L \eta u_{0} h^{*}}$ & 0.2487 & 0.1999 \\
\hline
\end{tabular}

Clearly, the flow within the experiments is laminar, and viscous forces are far greater than inertial forces. The Modified Reynolds Number shows that the viscous force in the direction of the gap is greater than the inertial force, therefore indicating that the essential flow behavior results from the same physical cause. This justifies a cross-scale comparison. The values of $N$ (in the range of 0.25 ) indicate that hydrodynamic friction is dominant in both experimental setups. Finally, the similar values of $N$ indicate that the nature of the hydrodynamic lubrication is similar across the two experiments. In conclusion, the comparison of the factors from the two friction cases shows that the experiments are in similar friction regimes, and that a cross-scale analysis of the flow is promising.

\section{Results}

The results of the macroscale experiments show trends that are very well studied on the microscale. It is possible to identify the influence of friction parameters such as velocity, viscosity and surface roughness on the Stribeck curve and its corresponding friction regime. This is used to underline the validity of the WDI for macroscale friction experiments. The results are shown in detail below. The full results can be accessed through Supplementary Materials associated with this paper.

\subsection{Coefficient of Friction}

In a filling pre-test, the amount of fluid needed to fully fill the gap height was determined. The topography was also considered during the pre-test. It was found for the glass 523R that $20 \mathrm{~mL}$ yields a state in which the gap height is nearly fully filled with fluid. This amount of lubricant is topography-specific. Figure 13 shows the COF over the rotational speed for the topography 523R for a lubricant amount of $20 \mathrm{~mL}$ (compare Figure 14). For the ornamental glass 523R, seven different measurement repetitions are shown, together with average values and standard deviations. The results show little variance and have a good repeatability. In the tests, the COF exhibits a distinctive behavior: It has a local maximum of 0.27 at the lowest speed. The COF decreases with increasing speed to a minimum of 0.23 at $4 \mathrm{rpm}$. Due to the rotational nature on the WDI, the contacts toward the inner radius of the disk move with a much lower relative velocity. This may lead to an overall increase of the COF. When the speed is further increased, the COF increases linearly to a maximum of 0.37 . This friction behavior has strong similarities to the Stribeck curve. It is supposed that this behavior corresponds to the transition from Mixed Lubrication to Hydrodynamic Lubrication, as indicated on the Stribeck curve shown in Figure 13 (right). Consequently, the friction regimes from the Stribeck curve can be associated with the presented results. The local maximum at $1 \mathrm{rpm}$ corresponds to boundary lubrication with dominant solid body contact; the minimum is at a state of mixed lubrication. At higher speeds, the regime is largely hydrodynamic lubrication. When evaluating these figures, the high viscosity of the lubricant has to be taken into account. 

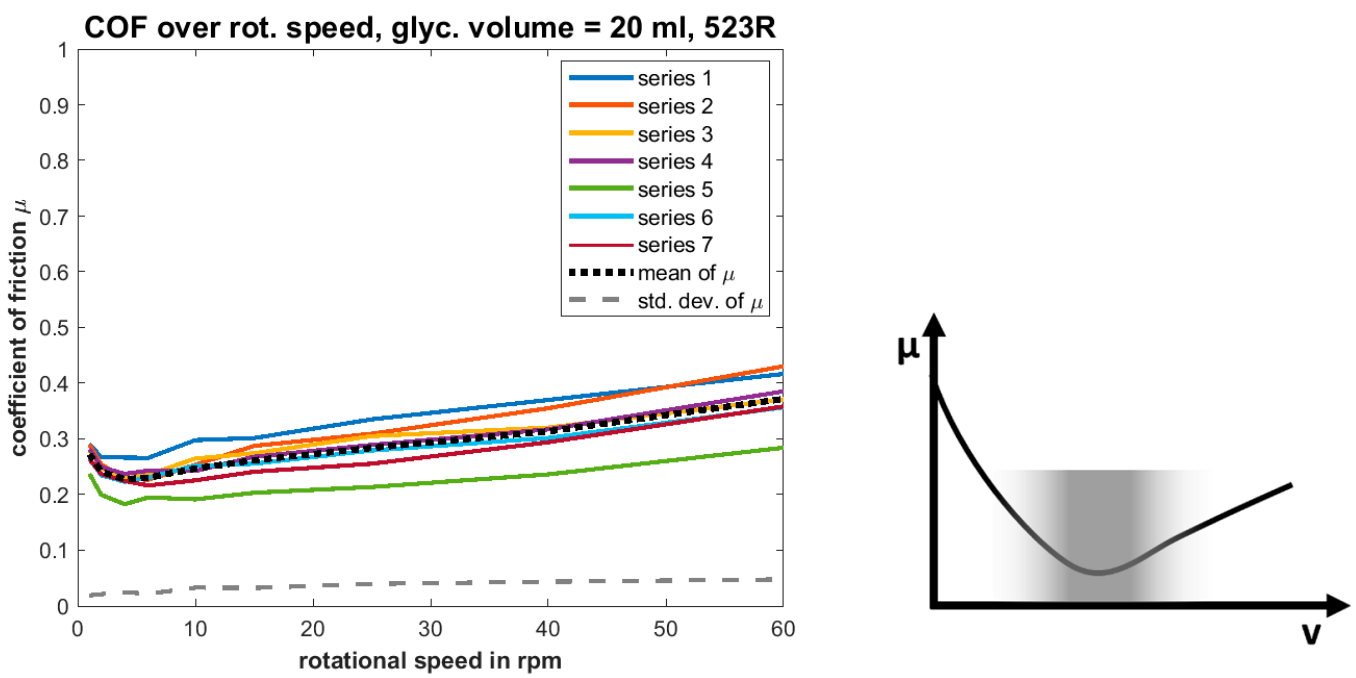

Figure 13. Coefficient of friction over the rotational speed at a glycerol volume of $20 \mathrm{~mL} 523 \mathrm{R}$.

The same measurement procedures were carried out using topography 528R whose surface topography has a significantly lower roughness than 523R, leading to a smaller gap height between the disks. This smaller gap height results in a decrease in the overall fluid volume compared to the previous study. Figure 14 shows the two contact partners with $20 \mathrm{~mL}$ (left) and $10 \mathrm{~mL}$ (right) (bottom up view with the WDI camera). The different coloring is a result of the dyes used. It can be observed that the global filling level of nearly $100 \%$ is similar for both glasses.

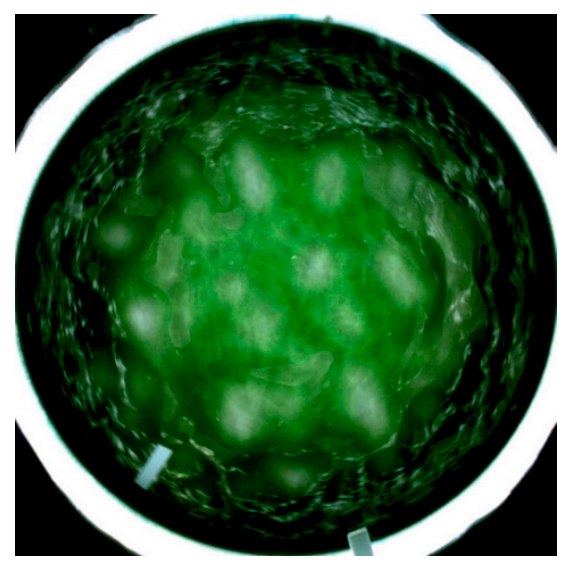

(a)

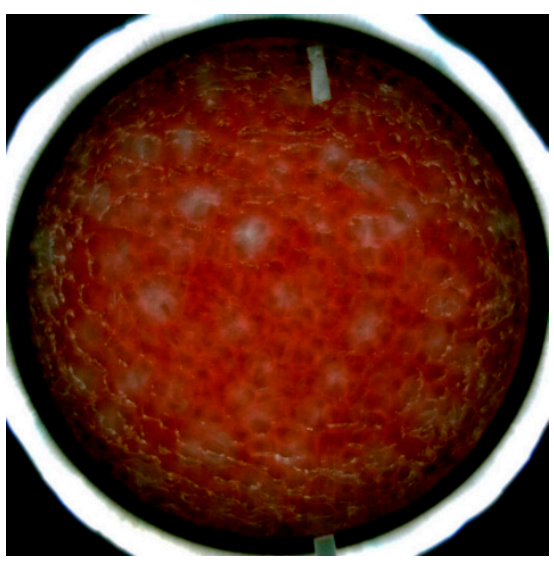

(b)

Figure 14. Contact partners in fully filled state (a) glass 523R with $20 \mathrm{~mL}$ (b) glass 528R with $10 \mathrm{~mL}$.

Figure 15 shows the COF over the rotational speed for topography 528R with a lubricant amount of $10 \mathrm{~mL}$. In contrast to Figure 13, the COF increases steadily with increasing speeds, with an overall maximum value of 0.7 . The highly viscous lubricant can be understood as the source of this high value. The linear nature of this increase is an indicator that this friction regime is predominantly hydrodynamic lubrication. Apparently, reduction of the surface roughness and of the gap height led to a shift of the associated friction regime towards the right side of the Stribeck curve (see Figure 15, grey area in the Stribeck curve). However, influences such as other topographic properties may distort this behavior. 
COF over rot. speed, glyc. volume $=10 \mathrm{ml}, 528 \mathrm{R}$

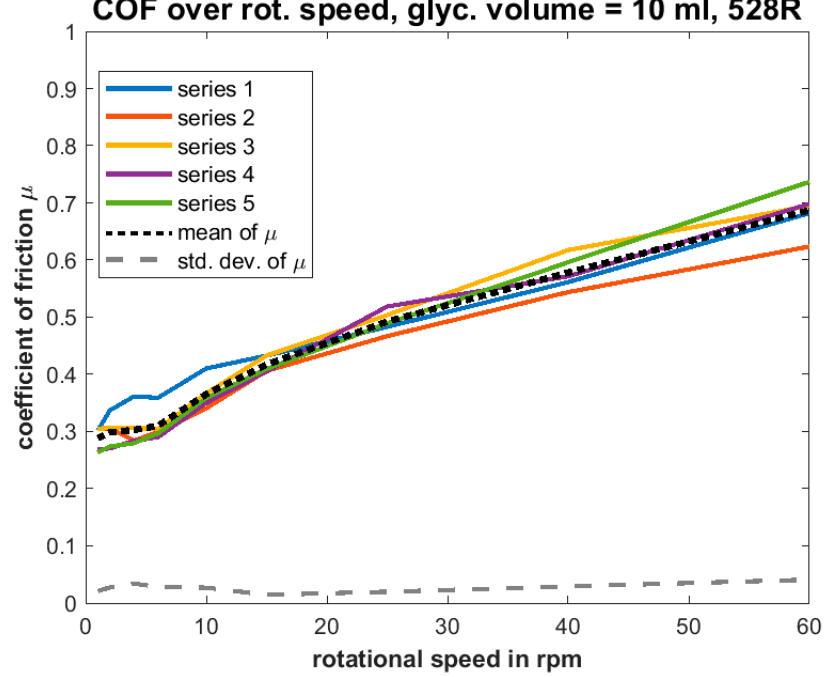

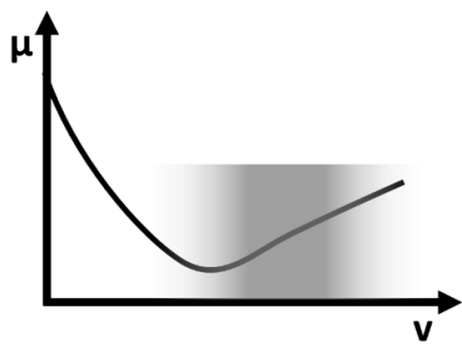

Figure 15. Coefficient of friction over the rotational speed at a glycerol volume of $10 \mathrm{~mL} 528 \mathrm{R}$.

From this parameter setup, the viscosity of the lubricant was modified by mixing the glycerol with water. Table 5 shows the different mixtures [21] and the associated dynamic viscosities.

Table 5. Lubricant mixtures between glycerol and water [21].

\begin{tabular}{cccc}
\hline \multirow{2}{*}{ Name } & \multirow{2}{*}{ Glycerol Weight Percentage in $\%$} & \multicolumn{2}{c}{ Viscosity $\boldsymbol{\eta}$ in Pa.s } \\
\cline { 3 - 4 } & & $\mathbf{2 0}{ }^{\circ} \mathbf{C}$ & $\mathbf{2 5}{ }^{\circ} \mathbf{C}$ \\
\hline Glyc. & 99.5 & 1.337 & 0.856 \\
M1 & 86 & 0.122 & 0.0926 \\
M2 & 63 & 0.01343 & 0.01083 \\
\hline
\end{tabular}

The mixtures were created in order to enable tests with a variety of viscosities. The viscosities of the mixtures differ by about one order of magnitude-the viscosity of M1 is one tenth that of glycerol and the viscosity of M2 is one hundredth that of glycerol. The viscosity exhibits a strong correlation to the fluid temperature. The average temperature of the lubricant was measured to be approximately $22{ }^{\circ} \mathrm{C}$. Due to low load and short load times, no significant heating of the fluid was detected. When varying the viscosity, precise measurements of the viscosity were not crucial, but rather the trend that the viscosity shows on the macroscale contact.

Figure 16 shows the COF over the rotational speed for the topography 528R with the M1 lubricant amount of $10 \mathrm{~mL}$ (nearly fully filled). The reduction of the viscosity changes the friction regime of the contact. This measured data exhibits a similar behavior to the data set from Figure 9 (523R and glycerol). A minimum COF can be found at $6 \mathrm{rpm}$. For lower speeds, the COF increases, which may be caused by increasing solid body contact. For higher speeds, the COF increases linearly, which is typically caused by hydrodynamic lubrication. This leads to the conclusion that, for this friction setup, the reduction of the roughness and of the gap height while also reducing the lubricant viscosity enabled the friction regime to remain nearly unaltered (compare estimated regime in the Stribeck curve on the right). 

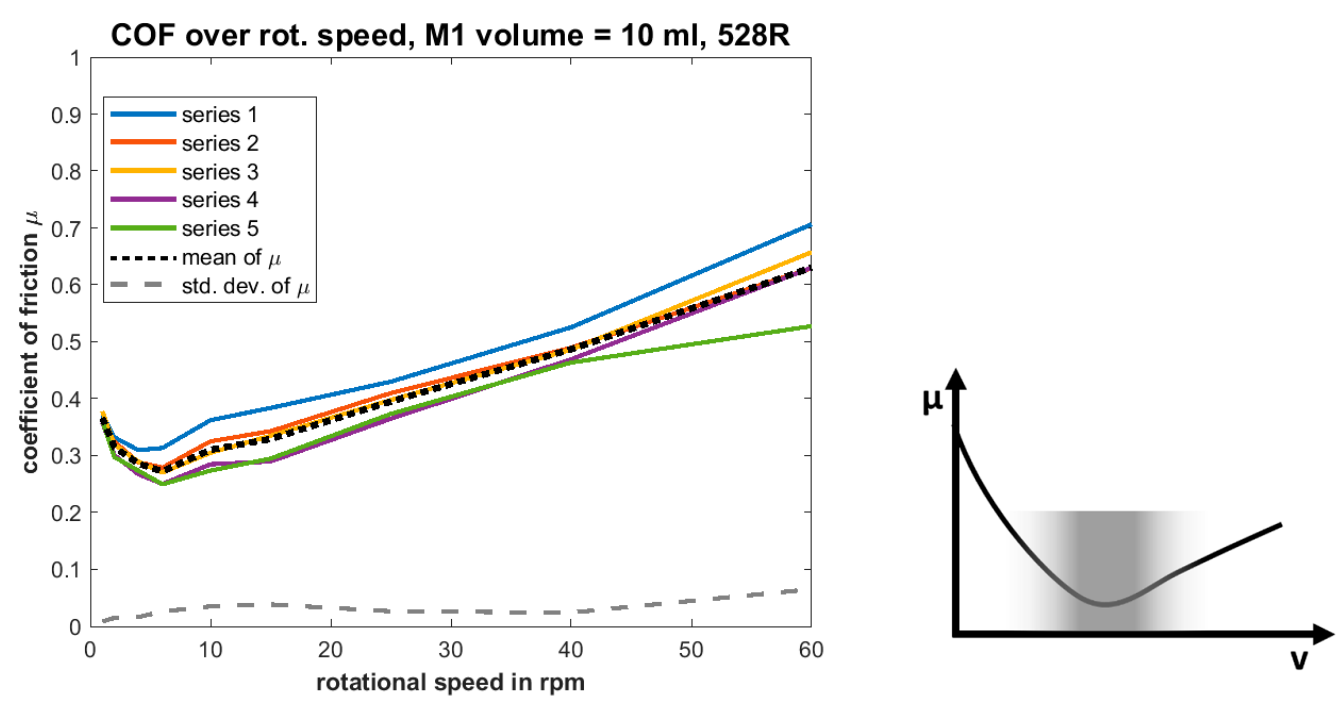

Figure 16. Coefficient of friction over the rotational speed at a M1 volume of $10 \mathrm{~mL} 528 \mathrm{R}$.

Figure 17 shows the COF over the rotational speed for the topography 528R with the M2 lubricant amount of $10 \mathrm{~mL}$. For this configuration, the COF is very high over all speeds. During this measurement procedure, vibrations and noise emissions from the WDI were stronger than in the other configurations. Together with the high COF, this is indicative of a high proportion of solid body contact. This leads to the supposition that this friction state is in the mixed lubrication regime with solid body contact. On the Stribeck curve, this corresponds to the leftmost area. With these results, the functionality of the test stand for fully filled gaps is verified.
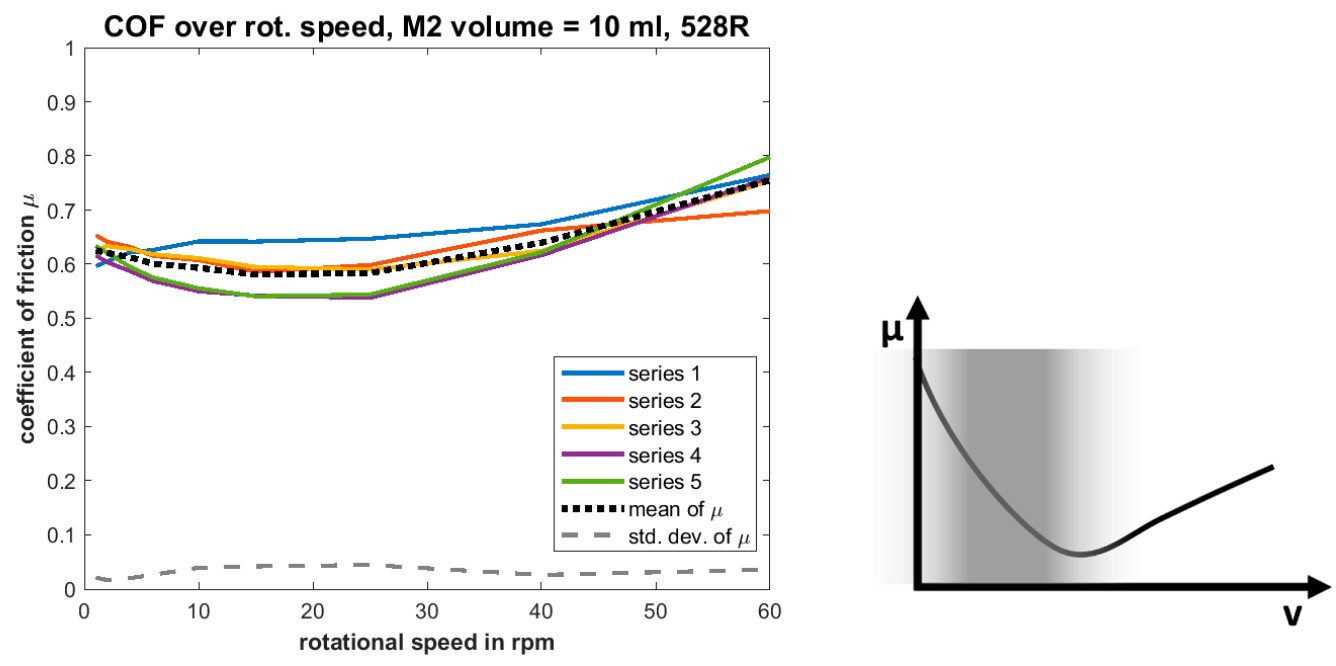

Figure 17. Coefficient of friction over the rotational speed at a M2 volume of $10 \mathrm{~mL} 528 \mathrm{R}$.

The results shown above all feature a fully filled gap. For all configurations, the fluid volume was also varied, as shown in Figure 9. The fluid volume was varied from $0 \mathrm{~mL}$ to $20 \mathrm{~mL}$ (523R)/ $0 \mathrm{~mL}$ to $10 \mathrm{~mL}$ (528R). These represent states with a dry gap to a fully filled gap, respectively. The studies aim to show a distinct relation between the $\mathrm{COF}$ and the filling level with good repeatability.

Figure 18 shows the COF over the filling level at $60 \mathrm{rpm}$. When there is no fluid in the contact, dry friction takes place. This correlates with a very high COF of 0.82 . When adding small amounts of fluid, the COF decreases significantly to a minimum of about 0.27. Adding additional fluid increases the hydrodynamic friction and the COF. Comparing the influence of the rotational speed to the influence of the filling level, there are considerable, qualitative similarities. Both show a distinct minimum of the $\mathrm{COF}$ followed by an increase, for low and high values of the varied parameter. For high rotational 
speeds and high filling levels, the COF rises linearly. This leads to the conclusion that for these configurations, an increase of the speed has a similar effect as an increase of the filling level. It was thus possible to investigate the transition from dry friction to hydrodynamic lubrication with the new test stand with very good repeatability. This indicates a great potential to investigate lubricant flow with the WDI (compare Section 3.2).

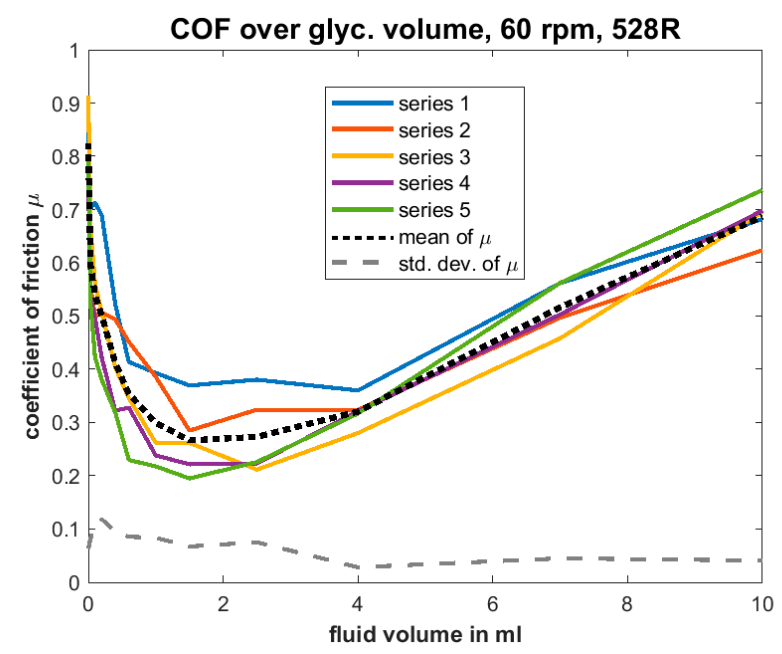

Figure 18. Coefficient of friction over the glyc. volume at $60 \mathrm{rpm} 528 \mathrm{R}$.

Comparing Figures 15 and 18 also yields interesting findings regarding the non-dimensional Stribeck parameter. Originally (compare Figure 1), halving the speed and halving the viscosity should lead to the same COF. Assuming a scalable viscosity with $\eta=\theta \cdot \eta_{\text {fluid }}$, halving the filling level will be similar to halving the viscosity. However, the COF at $10 \mathrm{~mL}$ and $60 \mathrm{rpm}$ is 0.69 . Halving the speed leads to COF of 0.52 , however halving the fluid volume (which will roughly halve the filling level) leads to 0.39. Although these are qualitative values from the WDI experiments, this is an indication that the interaction of filling level and viscosity is more complex than the common approach.

Figure 19 shows a detailed view of the results from Figure 18 at low filling levels. Here, it is worth noting that the filling level has significant effects, even at very small values. At $0 \mathrm{~mL}$ lubricant, dry friction takes place. The smallest amount of lubricant added was $0.03 \mathrm{~mL}$, corresponding to a filling level of $0.3 \%$. Adding this small amount of lubricant already decreases the COF to about 0.6 . This leads to the conclusion that the friction system is very sensitive to the amount of lubricant in the regime of dry/boundary friction.

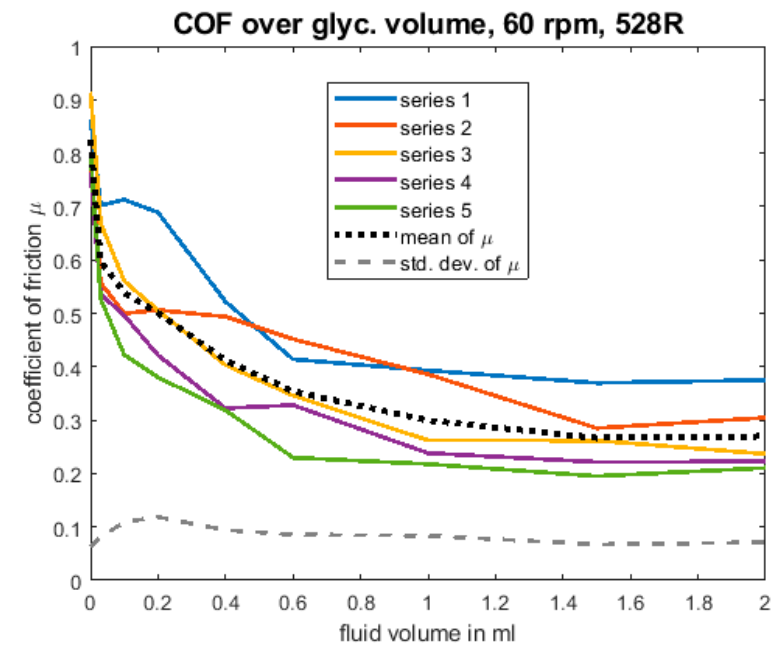

Figure 19. Coefficient of friction over the glyc. volume at $60 \mathrm{rpm} 528 \mathrm{R}$. 
Figure 19 also exhibits similarities to the HLT experiments (Figure 4). The COF falls steeply with small increments of fluid and then reaches a level that is insensitive to further fluid addition. This first qualitative comparison is currently limited to small fluid volumes for the following reason. When more fluid is added, on the HLT the fluid can flow out of the friction track, while the fluid in the WDI is trapped within the contact area.

Finally, all the data can be used to generate an overview of the correlation between the filling level and the rotational speed. Figure 20 shows the mean COF over the filling level and the rotational speed for the 528R and the M1 lubricant. It was approximated that $10 \mathrm{~mL}$ represents a filling level of one. It is apparent that the COF is highest for low filling levels (dry friction), low rotational speeds and for the combination of high speeds and high filling levels. Between these maxima, there is an L-shaped area in which the COF is comparably low.

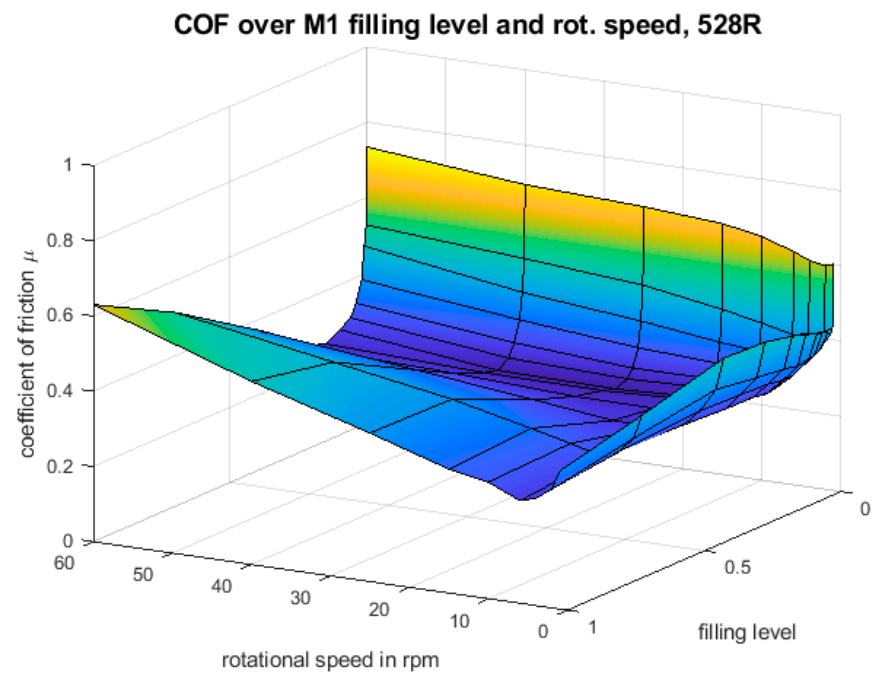

Figure 20. Coefficient of friction over the M1 filling level and the rotational speed 528R.

Additionally, the corresponding diagrams are also shown for the glyc. and M2 experiments in Figures 21 and 22 respectively.

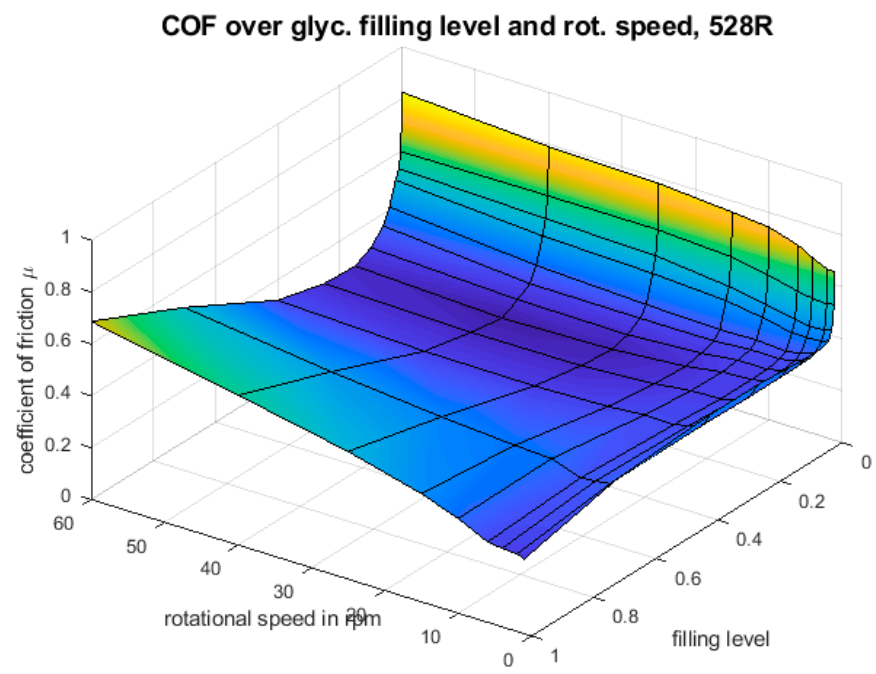

Figure 21. Coefficient of friction over the glyc. filling level and the rotational speed 528R. 
COF over M2 filling level and rot. speed, 528R

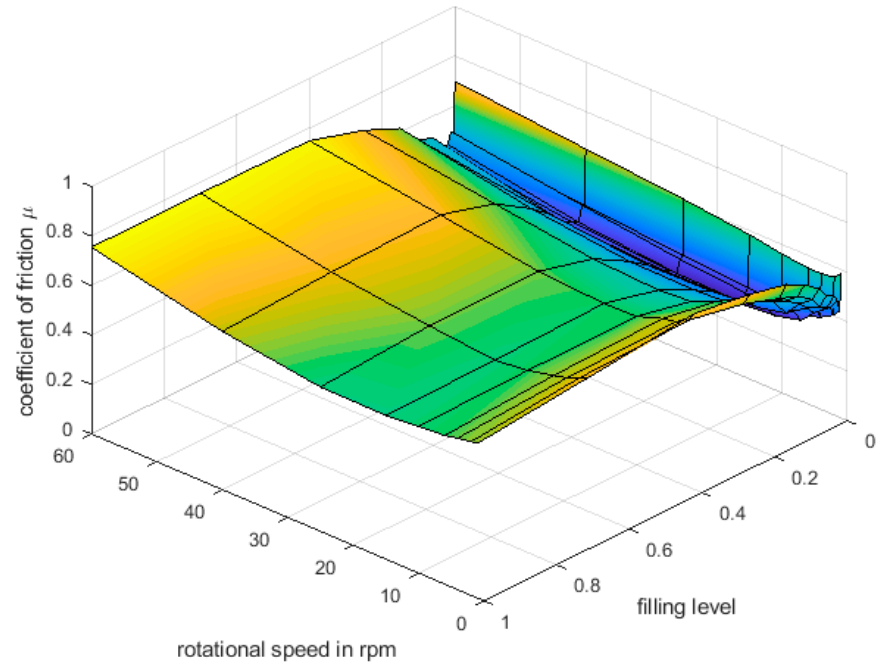

Figure 22. Coefficient of friction over the M2 filling level and the rotational speed 528R.

\subsection{Observations of Fluid Dynamics}

During the experiments, the fluid dynamics within the contact were closely monitored using the high definition camera system. The videos and images allowed for a more detailed look into the behavior of the fluid. Figure 23 shows multiple still images of the fluid state for different measurement configurations. All configurations feature the 528R topography. The images are arranged in such way that each column has the same speed, and each row the same lubricant. Each image was taken after the running-in process and at a filling level of 15\%. Initially, the fluid was evenly applied onto the disks. The numbers in the grey boxes show the corresponding COF for each picture. Figure 24 shows the corresponding COF over the angular velocity for the different lubricants.

Figure 23 shows a wide variety of different fluid behaviors. The top row contains images with the most viscous lubricant. The lubricant exhibits a distinctive pattern in which the fluid forms concentric rings. This pattern is best visible at $60 \mathrm{rpm}$. An analysis of the corresponding video shows that the rings largely form at the same radius and that the rings remain isolated, with no fluid exchange between them. The analyses also suggest that the rings form in close proximity to the asperities of the topography. Interestingly, it seems that the lubricant aggregates at the high-load contact points, rather than flowing to the valleys of the topography without contributing to the load carrying. The fluid performs a circular flow with half the rotational speed of the lower disk. The results show that centrifugal forces (such as in wet clutches, compare [11]) can be neglected due to the relatively small angular speed.

When decreasing the rotational velocity $(1 \mathrm{rpm})$, the rings spread out and cannot be clearly distinguished. The interchange of fluid between rings of varying radius is more distinct. When lowering the viscosity of the lubricant (e.g., M1, M2) the formation of the rings is shifted towards higher velocities. When looking at M2, the rings do not form below $60 \mathrm{rpm}$, and the thin layer does not occur at all. For lower speeds, the fluid spreads out into apparently randomly distributed islands. Here, very little fluid transport takes place. This state is characterized by predominant asperity contact. This is indicated by the high $\mathrm{COF}$ and the noise and vibration during testing.

Figure 24 shows the corresponding mean COF for the still images. It can be observed that the measurements with M2 have a greater COF, most likely due to the dominant solid body contact caused by the low viscosity of M2. 


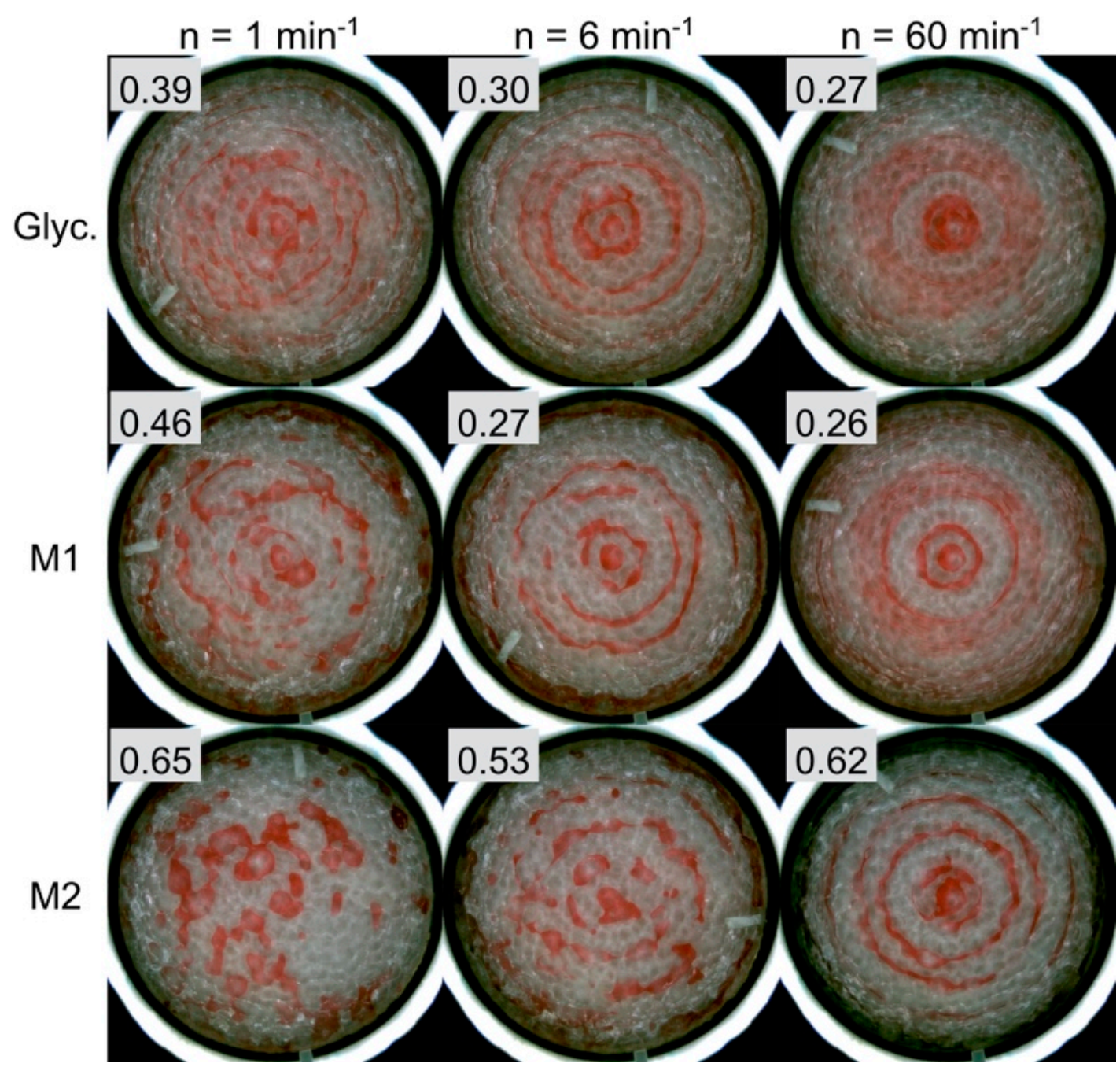

Figure 23. Snapshots of different fluid patterns in the contact at different angular velocities with different fluids.

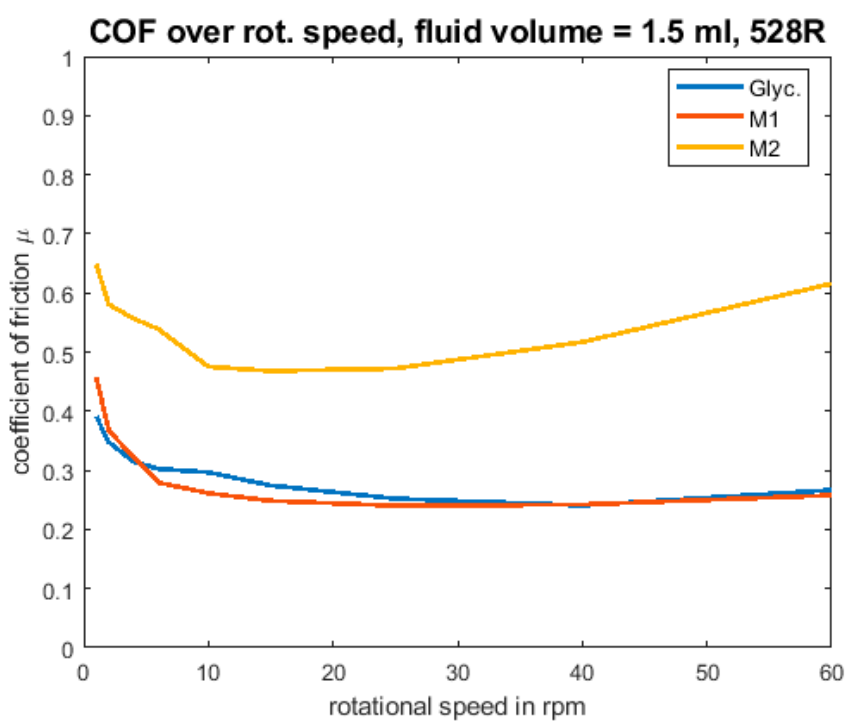

Figure 24. Coefficient of friction over the rotational speed at a fluid volume of $1.5 \mathrm{~mL} 528 \mathrm{R}$. 


\subsection{Gap Height}

Figure 25 shows the dynamic gap height increase over the rotational speed for a glycerol fluid volume of $10 \mathrm{~mL}$. Again, this gap height increase is calculated based on the differences between the mean height at the lowest speed and the mean height of the faster speeds. Therefore, all graphs start at $1 \mathrm{rpm}$ with $0 \mathrm{~mm}$ gap height increase. Overall, the measurements show a very good reproducibility. In the figure, it can be observed that the gap height rises with increasing speeds. At $60 \mathrm{rpm}$, the gap height increases by $0.9 \mathrm{~mm}$. The mean gap height was approximated by evaluating the topography and the supplied fluid in the gap. The gap height was estimated to be $0.5 \mathrm{~mm}$. In relation to this value, the mean gap height rises by $20 \%$. The rise in the gap height over the rotational speed follows a degressive trend. This well-known behavior can also be found in the literature [3].

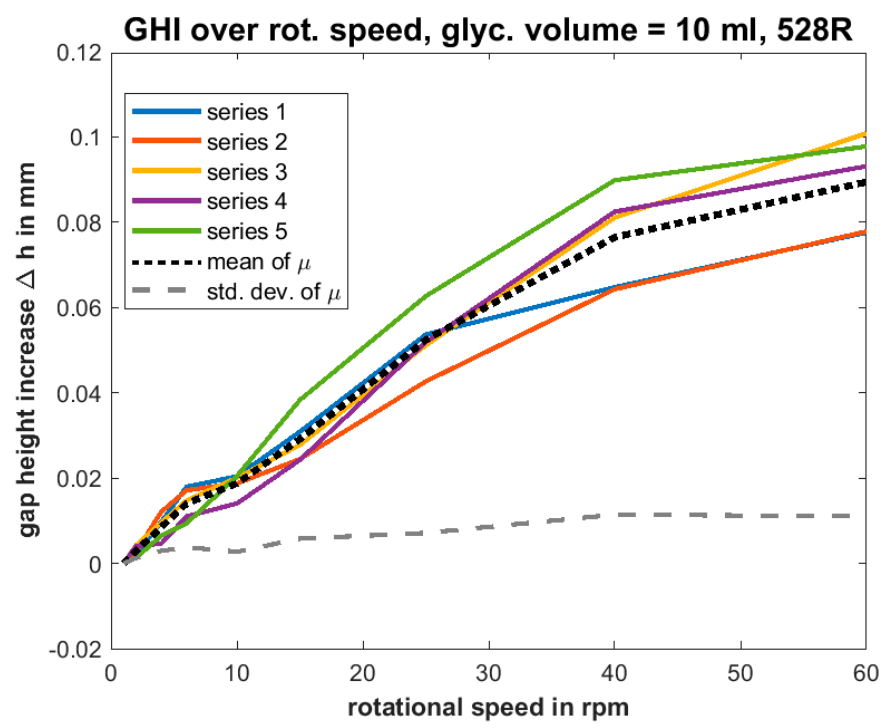

Figure 25. Gap height increase over the rotational speed at a glycerol volume of $10 \mathrm{~mL}$ 528R.

Figure 26 shows the dynamic gap height increase over the glycerol fluid volume for $60 \mathrm{rpm}$. The reference for the gap height is again the measurement with $1 \mathrm{rpm}$ and the same filling level. Therefore, this graph does not start at $0 \mathrm{~mm}$. It is noticeable that the gap height increase is an effect that predominantly takes place at higher filling levels (filling lever $>0.4$ ). The rise in the gap height over the fluid volume follows a progressive trend.

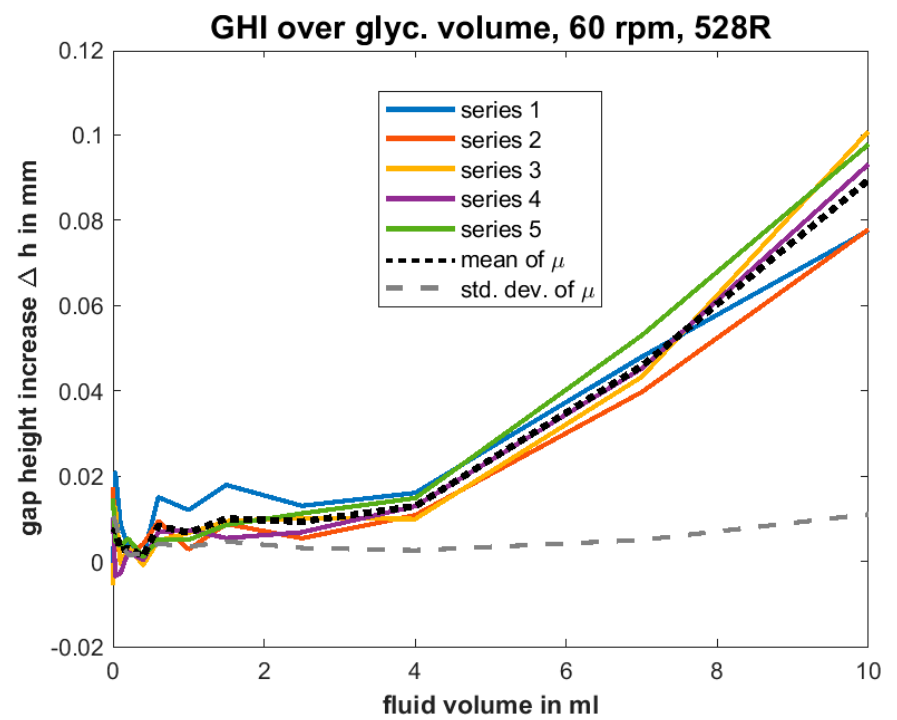

Figure 26. Gap height increase over the glycerol volume at $60 \mathrm{rpm} 528 \mathrm{R}$. 
The studies of the gap height also show very good repeatability. Therefore, the test stand is capable of performing such studies. Variations of the angular velocity have differing influences on the behavior than variations of the filling level. The cause of this will be the subject of future research.

\section{Conclusions}

Friction regimes with a partially filled gap are yet to be fully understood. In this research, a new approach that combines experiments on differing scales is presented. It was investigated whether the transition from dry friction to hydrodynamic lubrication can also be controlled through the filling level, and not only through the relative speed (Stribeck). In previous research, experiments were carried out using a conventional pin on disk tribometer setup. These experiments showed a distinct behavior between the COF and the fluid volume.

To enhance the understanding of the friction process in such a gap, a new experiment was carried out. In this paper, additional insights into the mechanics of the friction process were gained with a relatively simple macroscale experiment. The results show a typical Stribeck behavior and good repeatability. When varying the fluid volume, however, a similar correlation between the COF and the filling level was found. The results suggest that within boundaries the filling level may act as an active parameter to control the friction regime and the COF. While this seems obvious at first, there are some surprising quantities associated. The use of an effective viscosity (usually defined as the product of filling level and fluid viscosity) in the partially filled gap is to be viewed critically and the correlation between filling level and fluid flow seems to be more complex than commonly assumed. For example, halving the velocity leads to a different friction state than halving the fluid volume. This leads to the conclusion that the identification of the friction regime in starved lubrication should not only consider the pressure, the speed and the viscosity as independent parameters but also the filling level. This behavior is especially relevant for dry contacts that experience partial wetting. The findings are backed by additional observations of the mean gap height and the fluid behavior, and the experimental setup is a promising approach for understanding the partially filled friction regime.

With the combination of two different scales for the measurements, a first understanding of the tribological effects of lubricated contacts has been gained. This included the tribological behavior regarding the COF and the friction parameters such as the gap height. It was shown that the typical Stribeck behavior appears on both scales.

The considerations regarding scaling and the similar friction behavior of the micro and macroscale show that scaling seems to be a valid tool to investigate the fluid dynamics and their influence on the COF. With the still images from the contact zone, it was possible to determine characteristic fluid patterns that occur within the gap. The experiments show a complex interaction between the fluid behavior (e.g., rings, islands) and the COF. All measurements showed very good reproducibility with very low standard deviations.

The research between the two scales will be continued. In the future, the range of topographies will be extended, and a variation of the normal force can be included. With an enhancement of the measurement setup, prospective measurements can include a correlation between the contact dynamics and the current topographic condition. To investigate the flow of the lubricant, the deployment of tracer particles in the lubricant is planned. These will aid in measuring the flow of the fluid.

Supplementary Materials: The Supplementary Materials are available online at http:/ /www.mdpi.com/20754442/6/4/110/s1.

Author Contributions: Conceptualization, M.M. and G.-P.O.; Data curation, L.S.; Formal analysis, L.S.; Funding acquisition, M.M. and G.-P.O.; Investigation, L.S.; Methodology, M.M., L.S. and G.-P.O.; Project administration, M.M.; Software, L.S.; Supervision, M.M. and G.-P.O.; Validation, L.S.; Visualization, L.S.; Writing—original draft, L.S.; Writing-review \& editing, M.M.

Funding: This research was funded by "Deutsche Forschungsgemeinschaft (DFG)" project number 390252106 "Grundsatzuntersuchungen zu tribologischen Kontakten mit teilgefüllten Spalten" (Fundamental Studies on Tribological Contacts with Partially Filled Gaps (http:/ / gepris.dfg.de/gepris/projekt/390252106?language=en). 
Conflicts of Interest: The authors declare no conflict of interest. The funders had no role in the design of the study; in the collection, analyses, or interpretation of data; in the writing of the manuscript, and in the decision to publish the results.

\section{References}

1. Bhushan, B. Introduction to Tribology; John Wiley \& Sons: New York, NY, USA, 2013.

2. Heshmat, H.; Pinkus, O.; Godet, M. On a common tribological mechanism between interacting surfaces. Tribol. Trans. 1989, 32, 32-41. [CrossRef]

3. Heshmat, H. Tribology of Interface Layers; CRC Press: Boca Raton, FL, USA, 2010.

4. Wedeven, L.D.; Evans, D.; Cameron, A. Optical analysis of ball bearing starvation. J. Lubr. Technol. 1971, 93, 349-361. [CrossRef]

5. Jeng, Y.-R. Theoretical analysis of piston-ring lubrication Part II-Starved lubrication and its application to a complete ring pack. Tribol. Trans. 1992, 35, 707-714. [CrossRef]

6. Heshmat, H.; Artiles, A.; Pinkus, O. Parametric study and optimization of starved thrust bearings. In Fluid Film Lubrication, Osborne Reynolds Centenary (13th Leeds-Lyon Symposium on Tribology); Tribology Series; Elsevier: Amsterdam, The Netherlands, 1987; Volume 11, pp. 105-112.

7. Elrod, H.G. A computer program for cavitation and starvation problems. Cavitation Relat. Phenom. Lubr. 1974, 37, 37-41.

8. Müller, M.; Völpel, A.; Ostermeyer, G.-P. On the influence of fluid dynamics and elastic deformations on pressure buildup in partially filled gaps. Tribol. Int. 2017, 105, 345-359. [CrossRef]

9. Müller, M.; Jäschke, H.; Bubser, F.; Ostermeyer, G.-P. Simulative studies of tribological interfaces with partially filled gaps. Tribol. Int. 2014, 78, 195-209. [CrossRef]

10. Müller, M.; Ostermeyer, G.-P.; Bubser, F. A contribution to the modeling of tribological processes under starved lubrication. Tribol. Int. 2013, 64, 135-147. [CrossRef]

11. Neupert, T.; Benke, E.; Bartel, D. Parameter study on the influence of a radial groove design on the drag torque of wet clutch discs in comparison with analytical models. Tribol. Int. 2018, 119, 809-821. [CrossRef]

12. Bartel, D. Simulation von Tribosystemen; Vieweg und Teubner Verlag: Wiesbaden, Germany, 2010.

13. Hu, Y.Z.; Wang, H.; Guo, Y. Molecular dynamics simulation of Poiseuille flow in ultra thin film. Tribotest 1995, 1, 301-310. [CrossRef]

14. Washizu, H.; Ohmori, T. Molecular dynamics simulations of elastohydrodynamic lubrication oil film. Lubr. Sci. 2010, 22, 323-340. [CrossRef]

15. Chevalier, F.; Lubrecht, A.A.; Cann, P.M.E.; Colin, F.; Dalmaz, G. Film thickness in starved EHL point contacts. J. Tribol. 1998, 120, 126-133. [CrossRef]

16. Damiens, B.; Venner, C.H.; Cann, P.M.E.; Lubrecht, A.A. Starved lubrication of elliptical EHD contacts. J. Tribol. 2004, 126, 105-111. [CrossRef]

17. Cann, P.M.E.; Damiens, B.; Lubrecht, A.A. The transition between fully flooded and starved regimes in EHL. Tribol. Int. 2004, 37, 859-864. [CrossRef]

18. Müller, M.; Ostermeyer, G.-P. Measurements of partially lubricated contacts on different scales. PAMM 2017, 17, 629-630. [CrossRef]

19. Ostermeyer, G.P.; Perzborn, N. Dynamic Friction Measurements, Especially for High Power Applications; SAE Technical Paper; SAE: Warrendale, PA, USA, 2011.

20. Ostermeyer, G.-P.; Brumme, S.; Recke, B. The Wear Debris Investigator-A New Device for Studying the Formation of Particles in the Contact Area. In Proceedings of the Eurobrake Conference, Dresden, Germany, 2-4 May 2017.

21. D'Ans, J.; Lax, E. Taschenbuch für Chemiker und Physiker; Springer: Berlin/Heidelberg, Germany, 2013.

22. Reynolds, O. On the theory of lubrication and its application to Mr. Beauchamp tower's experiments, including an experimental determination of the viscosity of olive oil. Philos. Trans. R. Soc. Lond. 1886, 177, 157-234. [CrossRef]

23. Brodsky, E.E.; Kanamori, H. Elastohydrodynamic lubrication of faults. J. Geophys. Res. Solid Earth 2001, 106, 16357-16374. [CrossRef] 
24. Sahlin, F. CFD-Analysis of Hydrodynamic Lubrication of Textured Surfaces. 2003. Available online: http:/ / www.diva-portal.org/smash/record.jsf?pid=diva2\%3A1017947\&dswid=-8540 (accessed on 10 October 2018).

25. Hamrock, B.J.; Schmid, S.R.; Jacobson, B.O. Fundamentals of Fluid Film Lubrication; CRC Press: Boca Raton, FL, USA, 2004.

26. Han, J.; Fang, L.; Sun, J.; Wang, Y.; Ge, S.; Zhu, H. Hydrodynamic lubrication of surfaces with asymmetric microdimple. Tribol. Trans. 2011, 54, 607-615. [CrossRef]

27. Dowson, D. A generalized Reynolds equation for fluid-film lubrication. Int. J. Mech. Sci. 1962, 4, 159-170. [CrossRef]

28. De Kraker, A.; van Ostayen, R.A.; van Beek, A.; Rixen, D.J. A multiscale method modeling surface texture effects. J. Tribol. 2007, 129, 221-230. [CrossRef]

29. Brunetière, N.; Francisco, A. Multiscale Modeling Applied to the Hydrodynamic Lubrication of Rough Surfaces for Computation Time Reduction. Lubricants 2018, 6, 83. [CrossRef]

(C) 2018 by the authors. Licensee MDPI, Basel, Switzerland. This article is an open access article distributed under the terms and conditions of the Creative Commons Attribution (CC BY) license (http://creativecommons.org/licenses/by/4.0/). 